\title{
Integrative analysis identifies key molecular signatures underlying neurodevelopmental deficits in fragile $X$ syndrome
}

Kagistia Hana Utami ${ }^{1}$, Niels H. Skotte ${ }^{2}$, Ana R. Colaço ${ }^{2}$, Nur Amirah Binte Mohammad Yusof $^{1}$, Bernice Sim ${ }^{1}$, Xin Yi Yeo ${ }^{3,4}$, Han-Gyu Bae ${ }^{3}$, Marta Garcia-Miralles ${ }^{1}$, Carola I. Radulescu ${ }^{1}$, Qiyu Chen ${ }^{1}$, Georgia Chaldaiopoulou ${ }^{1}$, Herty Liany ${ }^{5}$, Srikanth Nama ${ }^{6}$, Prabha Sampath $^{6}$, Sangyong Jung ${ }^{3,4}$, Matthias Mann², Mahmoud A. Pouladi ${ }^{1,4,7, *}$

1 Translational Laboratory in Genetic Medicine, Agency for Science, Technology and Research (A*STAR), 8A Biomedical Grove, Immunos, Singapore 138648

${ }^{2}$ Novo Nordisk Foundation Center for Protein Research, University of Copenhagen, 2200 Copenhagen N, Denmark

${ }^{3}$ Singapore Bioimaging Consortium (SBIC), A*STAR, 11 Biopolis Way, Singapore 138667

${ }^{4}$ Department of Physiology, National University of Singapore, Singapore 117597

${ }^{5}$ Genome Institute of Singapore, Agency for Science, Technology and Research (A*STAR), 60

Biopolis Drive, 138672

${ }^{6}$ Institute of Medical Biology, Agency for Science, Technology and Research (A*STAR), 8A

Biomedical Grove, Immunos, Level 5, Singapore 138648

${ }^{7}$ Department of Medicine, National University of Singapore, Singapore 117597

*For correspondence: map@pouladilab.org (M.A.P.)

\section{Abstract}

Fragile $X$ syndrome (FXS) is an incurable neurodevelopmental disorder with no effective treatment. FXS is caused by epigenetic silencing of FMR1 and loss of FMRP expression. To investigate the consequences of FMRP deficiency in the context of human physiology, we established isogenic FMR1 knockout (FMR1KO) human embryonic stem cells (hESCs). Integrative analysis of the transcriptomic and proteomic profiles of hESC-derived FMRPdeficient neurons revealed several dysregulated pathways important for brain development including processes related to axon development, neurotransmission, and the cell cycle. We functionally validated alterations in a number of these pathways, showing abnormal neural rosette formation and increased neural progenitor cell proliferation in FMR1KO cells. We further demonstrated neurite outgrowth and branching deficits along with impaired electrophysiological network activity in FMRP-deficient neurons. Using isogenic FMR1KO hESC-derived neurons, we reveal key molecular signatures and neurodevelopmental abnormalities arising from loss of FMRP. We anticipate that the FMR1KO hESCs and the neuronal transcriptome and proteome datasets will provide a platform to delineate the pathophysiology of FXS in human neural cells.

\section{Introduction}

Fragile X syndrome (FXS) is the most common genetic cause of intellectual disability and autism spectrum disorder (ASD) [1]. In addition to cognitive impairment, individuals with 
FXS often exhibit seizures, hypersensitivity, impulsivity, and social anxiety [2]. FXS is caused by an expansion of CGG trinucleotide repeats in the 5' UTR of the FMR1 gene, located on chromosome $\mathrm{X}$, which results in specific hypermethylation of FMR1 and silencing of FMRP, its encoded protein [3]. FMRP, a brain-enriched RNA binding protein, has been shown to regulate the translation of as many as $800 \mathrm{mRNAs}$ in neurons by stalling ribosomes [4]. Loss of translational regulation of these mRNAs, whose products are involved in a wide range of neurodevelopmental and neuronal processes, is thought to underlie the pleiotropic molecular and clinical manifestation of FXS [5]. Despite continued therapeutic efforts and several clinical trials, no effective treatment has been developed to date [6].

The identification of individuals exhibiting a spectrum of FXS clinical phenotypes who carry mutations in FMR1 that disrupt FMRP's RNA binding activity have provided strong support for loss of function as the underlying cause of FXS [7-9]. This discovery further validated the use of FMR1 knockout $(\mathrm{KO})$ animal models to investigate the pathogenesis of FXS [10]. FMR1 KO mice exhibit a number of phenotypes reminiscent of symptoms seen in individuals with FXS such as enlarged testes (macroorchidism), increased susceptibility to seizures and sensory hypersensitivity, hyperactivity, as well as perseveration and repetitive behaviours [10]. A number of molecular and synaptic defects have also been identified in rodent models of FXS [11], including abnormalities in dendritic spine morphology [12], protein synthesis [13], and neurotransmission [14] which, combined with the neurological deficits, have paved the way for the discovery and interrogation of novel targets for therapeutic intervention [6].

Human pluripotent stem cells (hPSCs) have emerged in recent years as a powerful tool to overcome the inaccessibility of the brain and to explore the underlying mechanisms of neurological diseases [15]. Studies using hPSCs to model neurodevelopmental disorders such as Rett [16], Down [17], Angelman [18], and Timothy syndrome [19] have begun to elucidate the neurodevelopmental abnormalities and pathogenic mechanisms associated with these disorders in the context of human physiology. For FXS, studies using hPSC-derived neurons have begun to identify disease-associated defects including abnormal morphologies as well as aberrant synaptic function [20-26]. However, a caveat of studies published to-date is the use of FXS and control hPSC lines with different genetic backgrounds. Here, we describe the generation of isogenic FMR1 knockout (FMR1KO) human embryonic stem cell (hESC) lines and their use to investigate the pathophysiology of FXS in the context of human neural cells.

\section{Results}

\section{Generation of isogenic FMR1 knockout (FMR1KO) hESCs using CRISPR/Cas9}

Isogenic pluripotent stem cells are important tools to model genetic disorders in the context of a common genetic background while working in cell types of interest. To generate FMR1KO hESCs, we utilized CRISPR/Cas9 nucleases targeting exon 3 of the FMR1 gene in the male H1 hESC line (Figure S1a). We first evaluated the on-target activity of the FMR1-targeting sgRNAs in HEK293 cells using the Surveyor assay and confirmed cleavage for both sgRNAs (Figure S1b). Following electroporation of plasmids into the control hESCs, clones were screened for indels in FMR1 using the Surveyor assay (Figure S1c). Eight clones with indels in FMR1 out of approximately 48 colonies (17\% efficiency) were obtained, of which two clones with 8 and 17 base pair (bp) deletions were selected for further characterization, based on their predicted amino acid truncation (Figure 1a, Figure 
S1d). PCR amplification for Cas9 in both FMR1KO lines showed absence of product at the expected size $(2,045 \mathrm{bp})$, indicating no integration of the Cas 9 transgene in the targeted clones (Figure S1e). We termed these two clones FMR1KO1 (8 bp deletion) and FMR1KO2 (17 bp deletion).

To characterize the FMR1KO hESC lines and further interrogate neural phenotypes underlying FXS, we included a previously described FXS hESC line $[27,28]$ in our assessments. Consistent with FXS hESCs, we observed no expression of FMRP in FMR1KO hESCs by immunoblotting using two different antibodies that bind to the N-terminal region and the $\mathrm{KH}$ domains of FMRP (Figure 1b, Figure S1f) and immunofluorescence imaging (Figure 1c). Furthermore, proteome MS analysis confirmed the absence of FMRP peptides in the

a

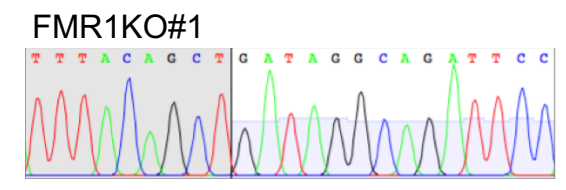

FMR1

5, ttgcttatttacagCTGGCAGCCTGATAGGCAGAT 3, (REF)

5, ttgcttatttacagCTG-..--.-ATAGGCAGAT 3, (FMR1K0\#1)

5, ttgcttatttacag-.-.-...-.-.-.AGAT 3, (FMR1K0\#2)

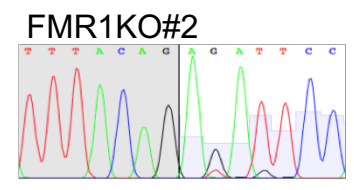

Exon 3

C
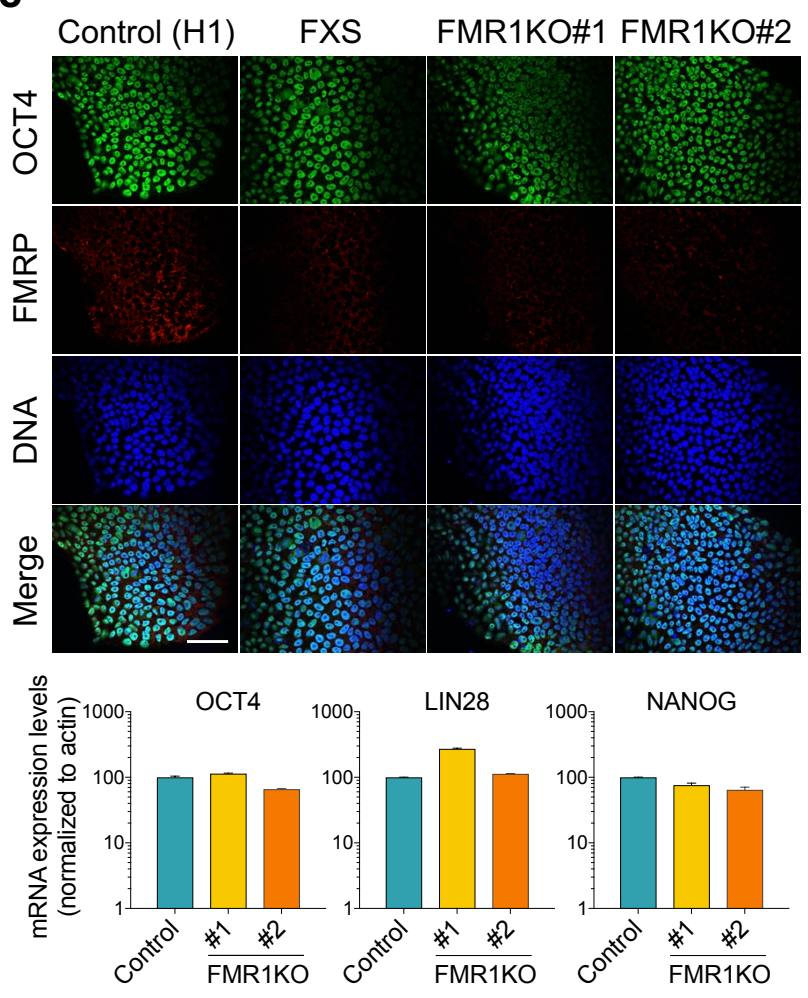

b

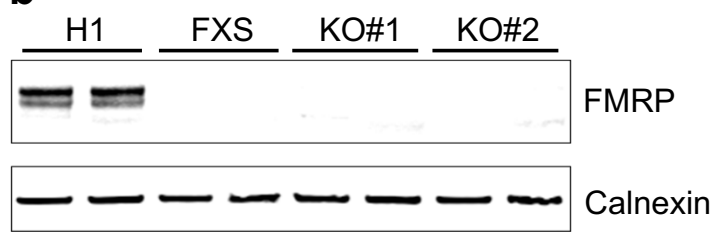

d

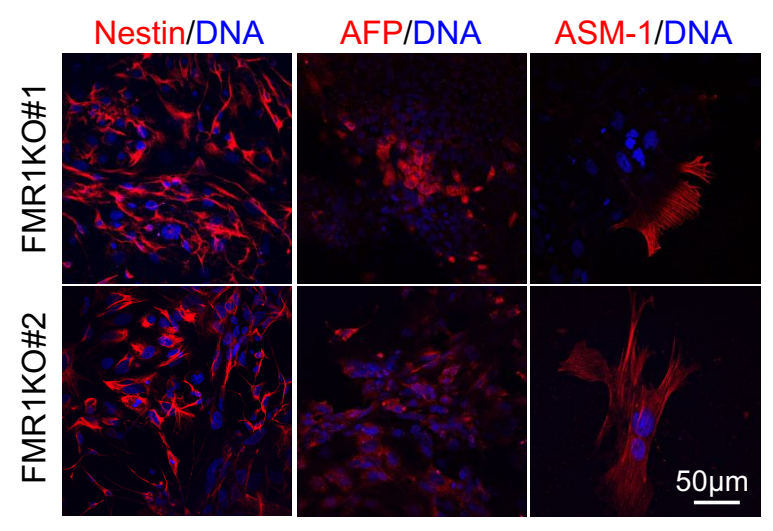

$\mathbf{e}$

FMR1KO\#1

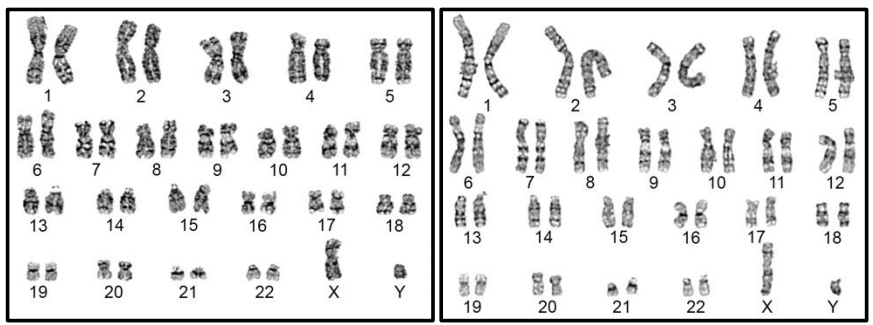

Figure 1. Generation of FMR1-/y H1 (FMR1KO) hESCs using CRISPR-Cas9 system. (a) Confirmation of indel and purity by Sanger sequencing. Schematic diagram showing the position of sgRNA sequence and indels generated in FMR1KO\#1 and FMR1KO\#2. (b) Immunoblot analysis showing absence of FMRP expression in FXS, FMR1KO1, and FMR1KO2. FMRP (80 kDa) and Calnexin (67 kDa). (c) FMR1KO hESCs express the indicated pluripotency markers as shown by immunostaining and qRT-PCR. Values shown as mean \pm SD, based on $\mathrm{n}=3$ replicates per genotype. (d) FMR1KO hESCs can give rise to three germ layers: ectoderm (Nestin), mesoderm (ASM-1), and endoderm (AFP). (e) FMR1KO hESCs maintained a normal karyotype demonstrated by G-banding analysis. 
FXS and FMR1KO lines (Figure S1g). Loss of FMRP did not appear to compromise the pluripotency of the isogenic FMR1KO hESCs as indicated by the uniform expression of pluripotency markers (OCT4, LIN28 and NANOG) (Figure 1c). We next examined the ability of isogenic hESC lines to differentiate into cells of the three germ layers via a standard embryoid body formation protocol. The ability to differentiate in vitro was confirmed by the presence of ectoderm (Nestin), mesoderm (atrial siphon muscle-1, ASM-1), and endoderm (alpha feto-protein, AFP) markers (Figure 1d). Importantly, no chromosomal aberrations were introduced by the gene targeting process and the FMR1KO hESCs maintained a normal karyotype (Figure 1e).

\section{Transcriptome and proteome analyses reveal cellular pathways altered in FMRP-deficient neurons}

To further investigate neural deficits caused by loss of FMRP, we differentiated control, FMR1KO, and FXS hESC into neurons using a previously described protocol [29] (Figure S2a). By day 37, flow cytometry analysis of the differentiated cultures, using an established panel of cell surface markers of neural and glial cells [30], showed that the proportion of neural versus glial cells derived from the different lines was comparable (Figure S2b). Further characterization of the resulting neuronal population showed them to be comprised mostly of MAP2/TUJ1-positive glutamatergic neurons ( $65-80 \%)$ with a lower proportion of GABAergic neurons ( 20\%) (Figure S2c,d).

To gain insights into the cellular processes disrupted by loss of FMRP, we performed RNA sequencing on day 37 neurons differentiated from control (H1) and isogenic FMR1KO hESCs, as well as FXS hESCs ( $\mathrm{n}=4$ for each cell line). Principal component analysis (PCA) of the transcriptional profiles showed tight clustering of biological replicates per genotype (Figure 2a). We identified a substantially higher number of differentially expressed genes (DEGs) in FMR1KO neurons versus isogenic control neurons compared with FXS versus control, as depicted in the volcano plots (Figure $\mathbf{2 b}, \mathbf{c}$ ). In total, we identified an overlap of 3,110 DEGs from FXS and FMR1KO neurons that were altered compared with controls, of which 1,525 genes were downregulated and 1,585 were upregulated (Figure 2d-g). Further statistical analysis with different threshold of log2 fold change reduced the number of DEGs substantially, indicating that most of the DEGs (83-97\%) had smaller fold changes $(|\log 2 \mathrm{FC}|>0)$. Separation of the differentially expressed genes by $\log 2$ fold change (FC) showed that $17 \%, 7 \%, 3 \%$ of the DEGs have a $|\log 2 \mathrm{FC}|>1,>1.5$, and $>2$, respectively (Figure 2f). Clustering of the 3,110 DEGs showed a high correlation between expression levels in FXS and FMR1KO samples supporting the current selection of genes of interest (Figure 2g). Functional annotation of DEGs shared between FMR1KO and FXS using gene ontology (GO) analysis and network visualization showed enrichment of a number of GO terms, including those related to neuron differentiation, and neurodevelopment, neurogenesis, neurotransmission for downregulated genes, and RNA processing and transport, translation, and cell cycle processes for upregulated genes (Figure 2h, Figure S3a). Among the neuronal differentiation GO categories, we identified genes involved in axon guidance, neurite outgrowth, and cell adhesion, such as DSCAM, GAP43, and PTPRT. To validate the changes between neuronal lines detected by RNA-seq, we confirmed these alterations by qRT-PCR (Figure S3b).

We compared our list of commonly identified genes in FXS and FMR1KO with two previously published transcriptome datasets of FXS neurons $[23,31]$, where we found a solid overlap of the identified genes (Figure S4a). From the shared genes between the three studies, we found that approximately $50 \%$ of our significantly regulated genes were 
bioRxiv preprint doi: https://doi.org/10.1101/606038; this version posted April 15, 2019. The copyright holder for this preprint (which was not certified by peer review) is the author/funder, who has granted bioRxiv a license to display the preprint in perpetuity. It is made available under aCC-BY-NC-ND 4.0 International license.

a

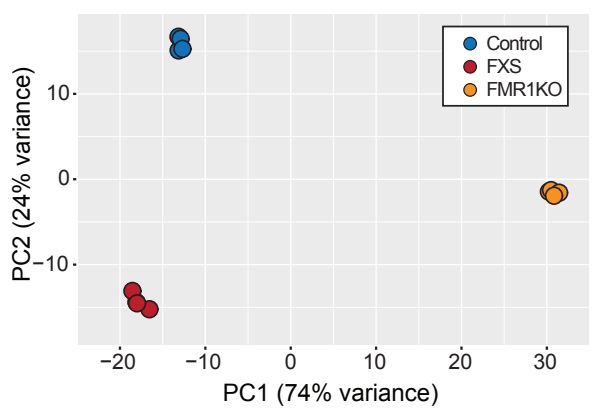

d

Down-regulated gene
Control vs. FMR1KO e

Up-regulated genes Control vs. FMR1KO

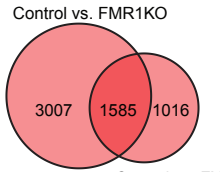

b

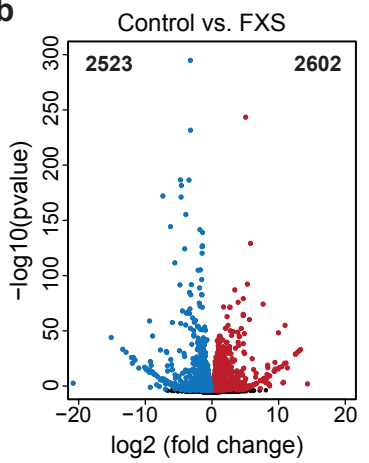

C

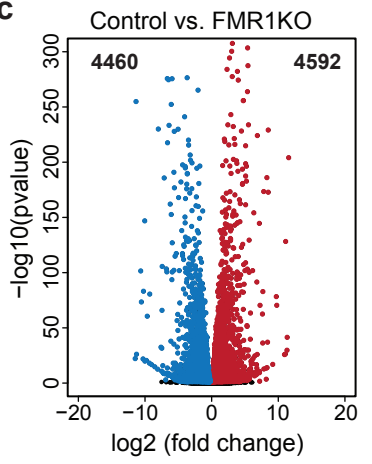

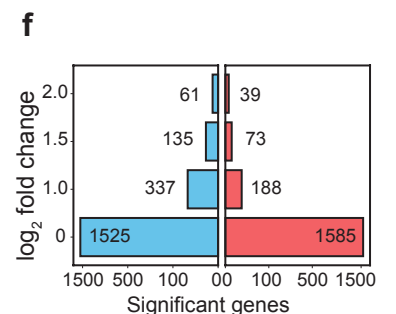
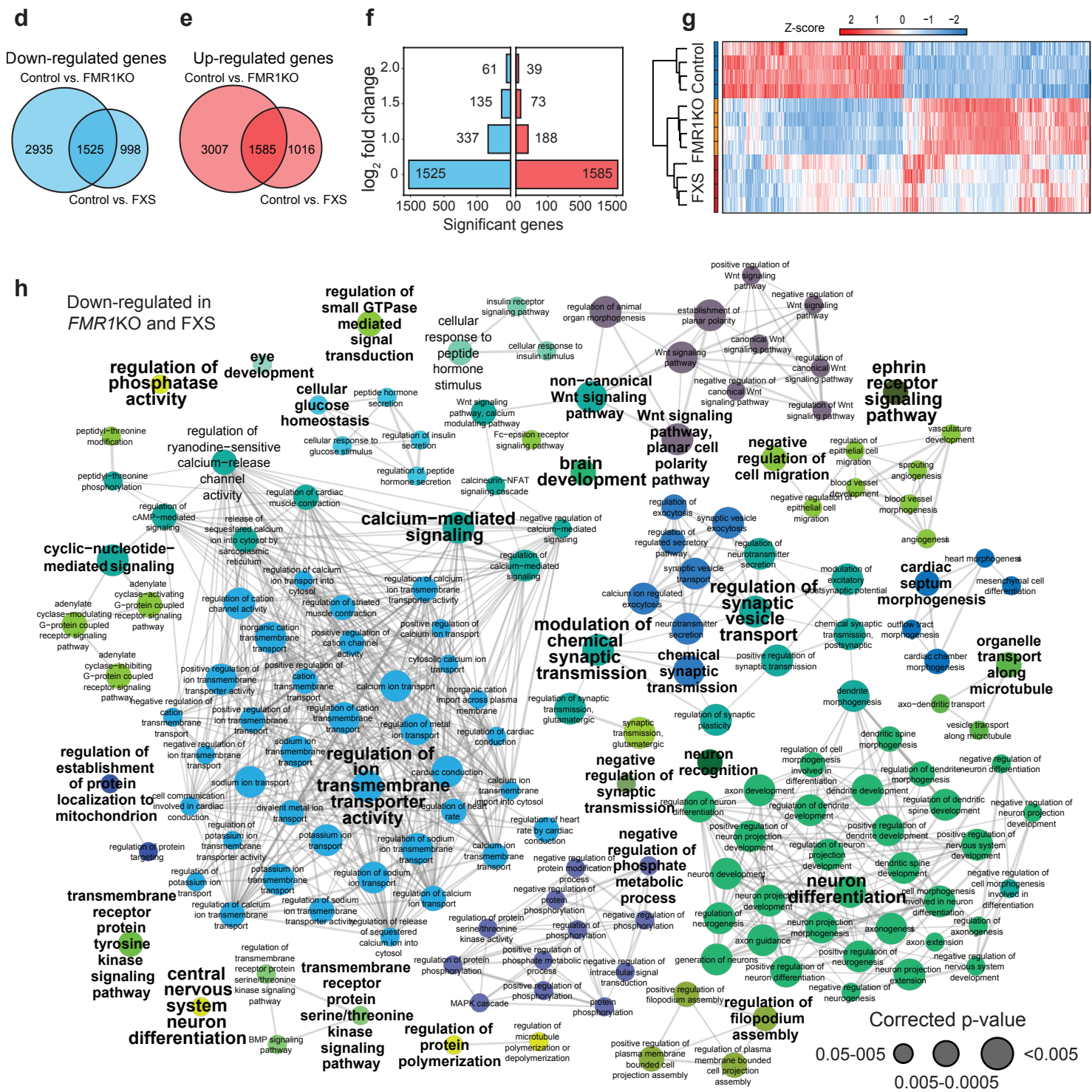

Figure 2. Global transcriptional changes in FMRP-deficient neurons. (a) Principal component analysis (PCA) shows tight clustering of replicates for each genotype; $(\mathbf{b}, \mathbf{c})$ Volcano plots of -Log10 ( $p$-value) versus the Log2 (fold change) of transcript levels for all genes. Relative to control, significantly downregulated genes are shown in blue and upregulated genes are shown in red; (d,e) Venn analysis showing genes that are similarly downregulated (d) and upregulated (e) between control vs FXS and control versus FMR1KO; (f) bar plot showing the number of significant changes dependent on the cut-off in log2 fold changes, (g) Heatmap of differentially expressed genes that are common to FXS and FMR1KO neurons; (h) Functional annotation of common downregulated genes in FXS and FMR1KO neurons; functional annotation of common upregulated genes can be found in Figure S3a. 
also regulated in either the Boland et al. or the Halevy et al. study. (Figure S4b and Table S2). Importantly, of our regulated DEGs, we found significant association with 239 Simons Foundation Autism Research Initiative (SFARI) genes, a database of genes linked to autism [32] (Figure S4c,d and Table S2) $\left(|\log 2 \mathrm{FC}|>0, \mathrm{p}=6.35 \times 10^{-10}\right.$ and $|\log 2 \mathrm{FC}|>1$, $\left.\mathrm{p}=7.82 \times 10^{-3}\right)$. We also observed significant association for DEGs with $|\log 2 \mathrm{FC}|>0$ for FMRP substrates $\left(246\right.$ genes, $\left.\mathrm{p}=1.1 \times 10^{-29}\right)$ (Figure S4c and Table S2). Together, our findings support that loss of FMRP results in transcriptional dysregulation, which alters processes involved in central nervous system development, such as neuronal differentiation, neurogenesis, and cell cycle regulation.

To map the FXS proteome, we collected neurons on day 37 of differentiation, the same time-point used for the transcriptome analysis, and subsequently profiled protein expression changes by MS analysis. To establish a baseline for the technical and biological quality, we first compared protein and peptide numbers and evaluated the reproducibility. From this, we identified a total of 5007 proteins, where 4210 were selected for further investigations after stringent filtering, and compared protein and peptide identification across the 3 genotypes (Figure S5a,b). The majority of the selected proteins in our study were shared between all three genotypes (Figure S5c). Furthermore, we observed strong reproducibility (Pearson correlations ranging from 0.97-0.98) between biological replicates (Figure S5d). The high reproducibility in our analysis was further supported by a PCA demonstrating a strong separation of the three genotypes (Figure 3a), supporting that our proteomic analysis provides a sound foundation for genotypic comparison. Next, we performed a system-wide comparison in more detail comparing FXS and FMR1KO to control, respectively. (Figure $3 \mathbf{b}, \mathbf{c}$ and Figure S5e,f). Similarly to the results from the RNA-seq statistical analysis, the vast majority of significantly regulated proteins depicts smaller log2 fold changes $(|\log 2 \mathrm{FC}|>$ 0 ) with only $17 \%$ and $13 \%$ of down- and upregulated proteins, respectively, showing log2 fold changes beyond $1(|\log 2 \mathrm{FC}|>1)$ (Figure S5e). A hierarchically-clustered heatmap shows grouping of the samples according to the expected genotypes, whereas column values suggest a closer correlation between FXS and FMR1KO expression levels than with expression levels in Control samples (Figure S5f). We identified several protein changes with a total of 577 and 2198 proteins exhibiting increased or decreased expression in FXS and FMR1KO compared to control, respectively, as depicted in volcano plot (Figure 3b,c).

Next, we performed pathway enrichment analysis for significantly regulated proteins in FXS (343 downregulated and 234 upregulated proteins) and FMR1KO (1247 downregulated and 951 upregulated proteins) samples, separately. In the same way as the RNA-seq data, the enriched GO terms for differentially expressed proteins in FMR1KO showed down-regulation of proteins involved in neuron development, neuron differentiation, neurotransmitter secretion, and regulation of calcium ion transport, whereas the upregulated proteins gave rise to GO terms including RNA processing and splicing and ribosome biogenesis. In addition, the GO terms for differentially expressed proteins in FXS showed enrichment in vesicle transport and synaptic signalling for downregulated proteins, and cell cycle processes, DNA replication and DNA metabolic processes for upregulated proteins. Among the downregulated proteins we observed several candidates such as CNTNAP2 [33,34], GPRIN3, KIF5C [35] and CNTN1 previously associated with FXS and other neurodevelopmental disorders as well as neurodegenerative diseases.

To identify and summarize the common changes between in FXS and FMR1KO, we performed pathway enrichment analysis for differentially expressed proteins shared between FXS and FMR1KO (244 downregulated and 141 upregulated proteins). We condensed the data and visualized the GO terms for biological processes (GOBP) terms as two networks representing up- and downregulated common protein changes (Figure S6a,b). Interestingly 
bioRxiv preprint doi: https://doi.org/10.1101/606038; this version posted April 15, 2019. The copyright holder for this preprint (which was not certified by peer review) is the author/funder, who has granted bioRxiv a license to display the preprint in perpetuity. It is made available under aCC-BY-NC-ND 4.0 International license.
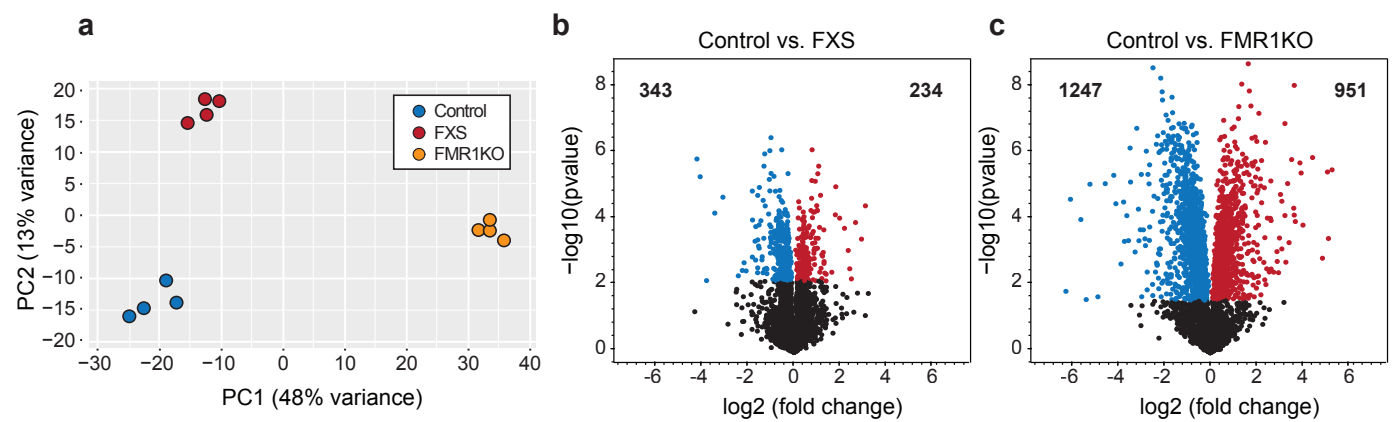

d
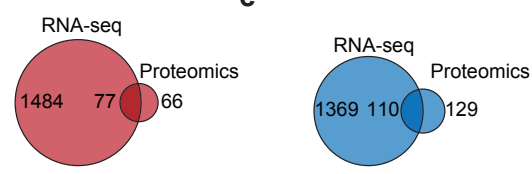

f
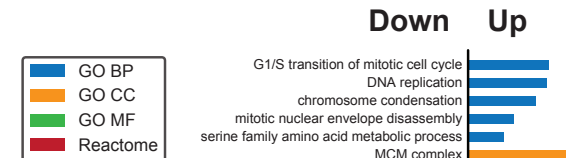

Down Up

Reactome

mitotic nuclear envelope disassem
serine family amino acid metabolic proces

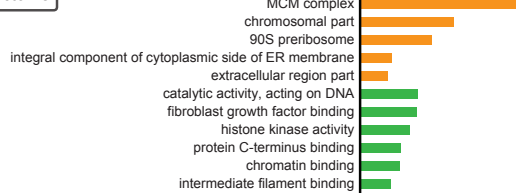

chromatin bindin Unwinding of DNA Condensation of Prometaphase Chromosomes
Growth Factor-2 mRNA Binding Proteins bind RNA Metabolism of amino acids and derivatives

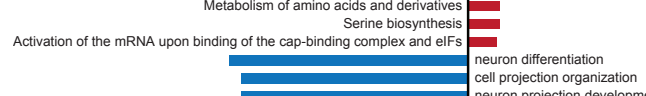

neuron projection development

\begin{tabular}{l|l} 
regulation of calcium ion-dependent exocytosis \\
neurotransmitter transport \\
system development
\end{tabular}

regulation of calcium ion-dependent exocytosis
neurotransmitter transport
system development

system development
Wnt signaling pathway, calcium modulating pathway

Wnt signaling pathway, calcium mod
establishment of vesicle localization

establishment of vell signaling

cell morphogenesis
second-messenger-mediated signaling protein localization to juxtaparanode region of ax
anatomical structure morphogenesis calcium-mediated signaling

ephrin receptor signaling pathway plasma membrane organization cell adhesion

Tegulation of homotypic cell-cell adhesion tation of positive chemotaxis

cell recognitio
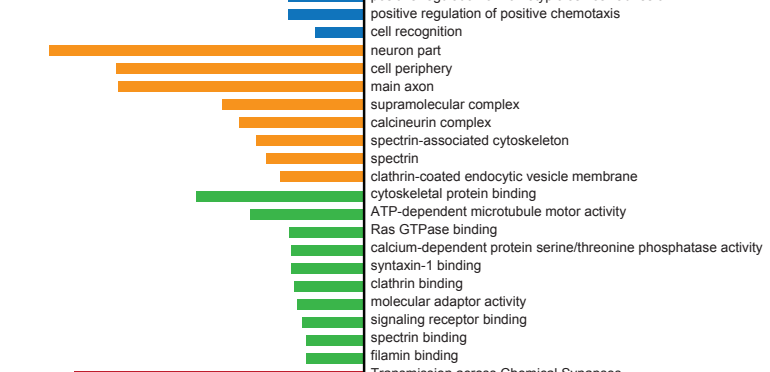

h
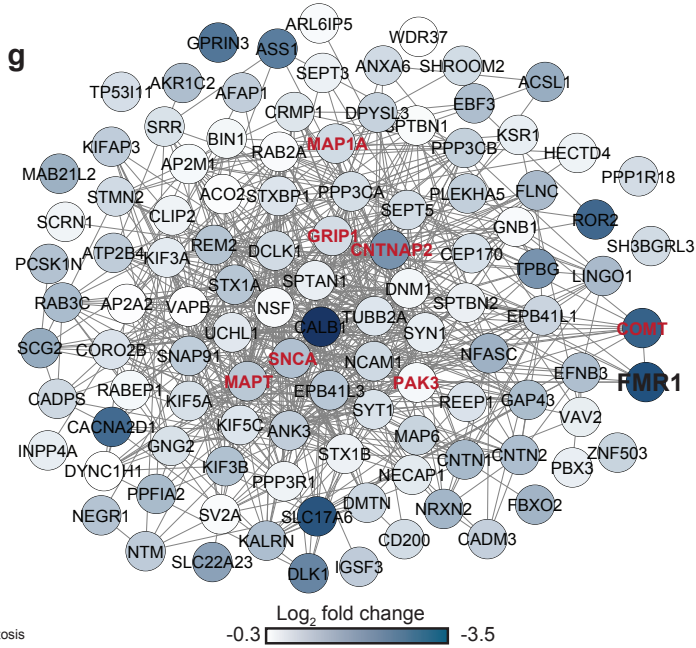

$-0.3 \lcm{\text { Log }_{2} \text { fold change }}-3.5$
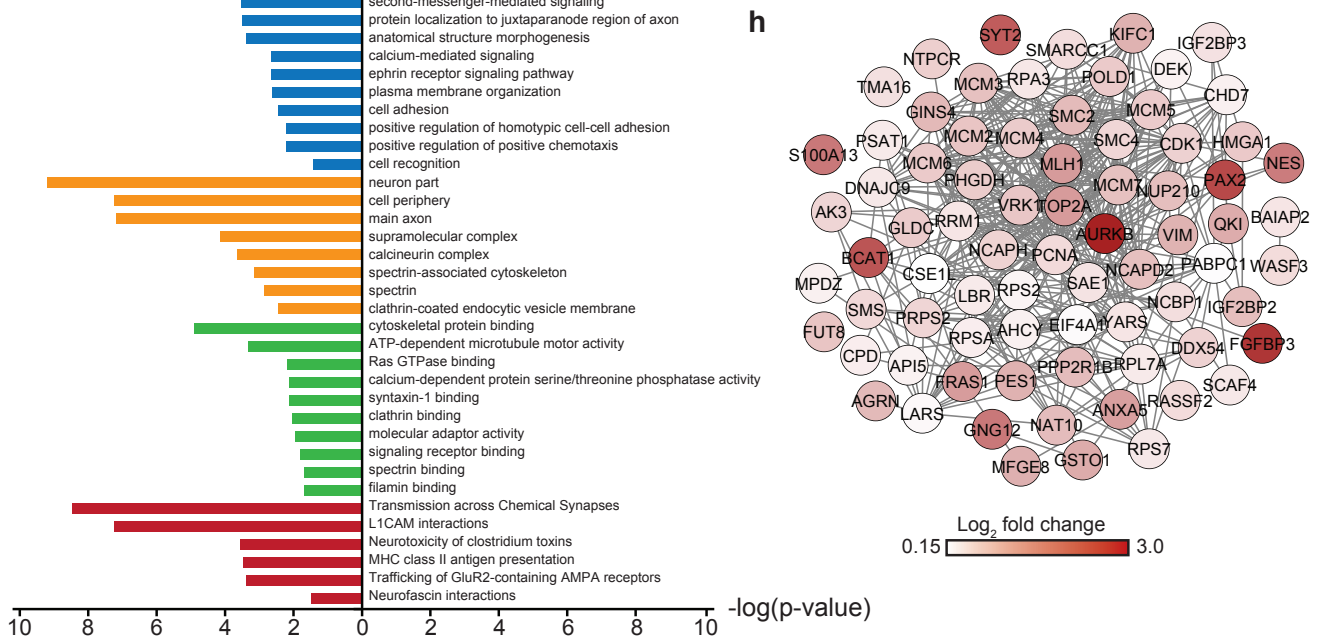

Figure 3. Global proteomic changes in FMRP-deficient neurons. (a) Principal component analysis (PCA) shows clustering of replicates for each genotype; (b,c) Volcano plots of -Log10 ( $p$-value) versus the Log2 (fold change) of protein levels for all proteins. Relative to control, significantly downregulated proteins are shown in blue and upregulated proteins are shown in red; (d,e) Venn analysis showing common significant changes shared between FMR1KO and FXS as well as between the RNA-seq and proteomics data. 77 upregulated proteins/genes (d) and 110 downregulated (e) proteins/genes; (f) Functional annotations for the significant common changes, where a subset (based on protein with lowest p-value per cluster group for GO terms BP, CC, and MF as well as Reactome) is displayed. (g,h) STRING protein-protein interactions network for the common significant upregulated $(\mathbf{g})$ and downregulated $(\mathbf{h})$ changes at both the RNA and protein level are illustrated. Proteins associated with FMR1 are highlighted in red. 
for the networks, we found cellular processes based on downregulated proteins related to neurotransmitter secretion, synaptic transmission, transport, and dendrite development and DNA replication, gene expression, and cell cycle processes for upregulated proteins. Importantly, the data reveal many GO categories similar to those identified in the transcriptional enrichment analysis, highlighting the complementary of the proteomic and transcriptional analyses and providing further evidence linking loss of FMRP to functional perturbations in these pathways. This notion is further supported by the enrichment of both SFARI and FMR1/FMRP related proteins (Figure $\mathbf{S 7 a}, \mathbf{b}$ ). In the proteomics data set, we found significant association with 40 SFARI database genes and 57 FMRP genes $(|\log 2 \mathrm{FC}|>0 ; \mathrm{p}=8.32$ $\times 10^{-3}$ and $\mathrm{p}=2.12 \times 10^{-7}$, respectively) (Figure S7a,b and Table S3), which demonstrated that our findings at the RNA level translated to the protein level providing another layer of functional insight. Collectively, our findings highlight several biological processes and pathways altered in FMRP-deficient neurons of potential relevance to the pathogenesis of FXS.

Lastly, to combine the knowledge gathered from the RNA-seq and proteomics analyses, we explored the overlap of significant changes between the two data sets (Figure S8a). First, we compared the expression correlation between the two data sets and found a good correlation between the RNA and protein expression $\left(\mathrm{R} 2=0.42 ; \mathrm{p}=8.6 \times 10^{-171}, \mathrm{R} 2=0.44 ; \mathrm{p}=9.9 \times\right.$ $10^{-190}$, and R2=0.42; $\mathrm{p}=2.9 \times 10^{-171}$, for control, FMR1KO, and FXS, respectively, Figure S8b). Notably, we found significant 187 genes and proteins in common between the two data sets, where 77 and 110 were significantly up- and downregulated, respectively (Figure 3d-e). The enrichment analysis on the common changes between the two data types (RNA-seq and proteomics) revealed several altered interesting pathways related to neurotransmitter transport, synaptic signalling, neuron differentiation, and brain development for down regulated proteins/genes (Figure 3f) as well DNA replication, mitosis, and cell cycle for upregulated proteins/genes. These findings clearly encapsulate the commonalities between the two data sets and demonstrate that the resources described in this manuscript can be used independently or in combination. Lastly, in order to better understand how these genes and proteins might interact with each other, we looked into protein-protein interaction networks using STRING database (string-db.org) (Figure $\mathbf{3 g}, \mathbf{h}$ ). The networks were plotted using the edge_betweenness clustering algorithm to identify highly connected nodes. This method clusters together proteins, which are known to cooperate and have correlating gene function annotations [36]. From this analysis, we found six genes (COMT, MAP1A, GRIP1, $P A K 3, S N C A, M A P T$, and CNTNAP2) to be closely associated with FMR1/FMRP. Interestingly, SNCA (alpha-synuclein), MAPT (Tau), CNTNAP2 (Contactin associated protein-like 2), PAK3 (P21 protein), and COMT (catechol-O-methyltransferase) have associations with Parkinson's disease, Alzheimer's disease, autism, intellectual disability, and schizophrenia, respectively. These findings were further supported by a search for curated gene-disease associations in DisGeNET database (www.disgenet.org). From our 187 common candidates, 53 downregulated and 28 upregulated genes were found to be associated with "Nervous System Diseases" and "Mental Disorders".

\section{Loss of FMRP leads to abnormal neural rosette formation and increased neural progenitor proliferation}

The transcriptional and proteomic analysis highlighted changes in neuronal development and neurogenesis; therefore, we sought to examine neural rosette formation following neural induction as a measure of early neurodevelopment in FMR1KO and FXS lines. We differentiated hESCs into neural rosettes using a previously described floating embryoid 
a
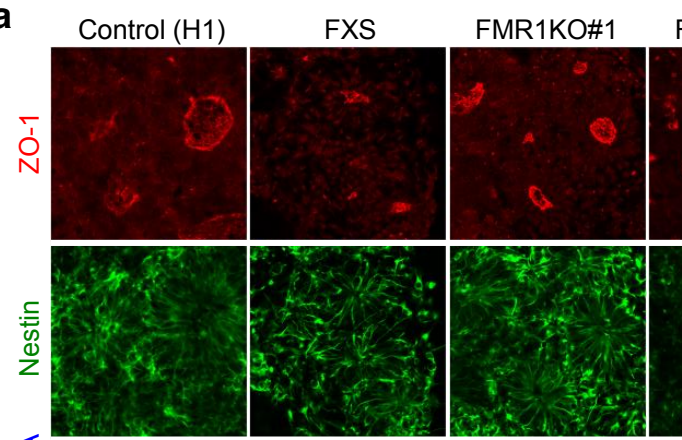

FMR1KO\#2
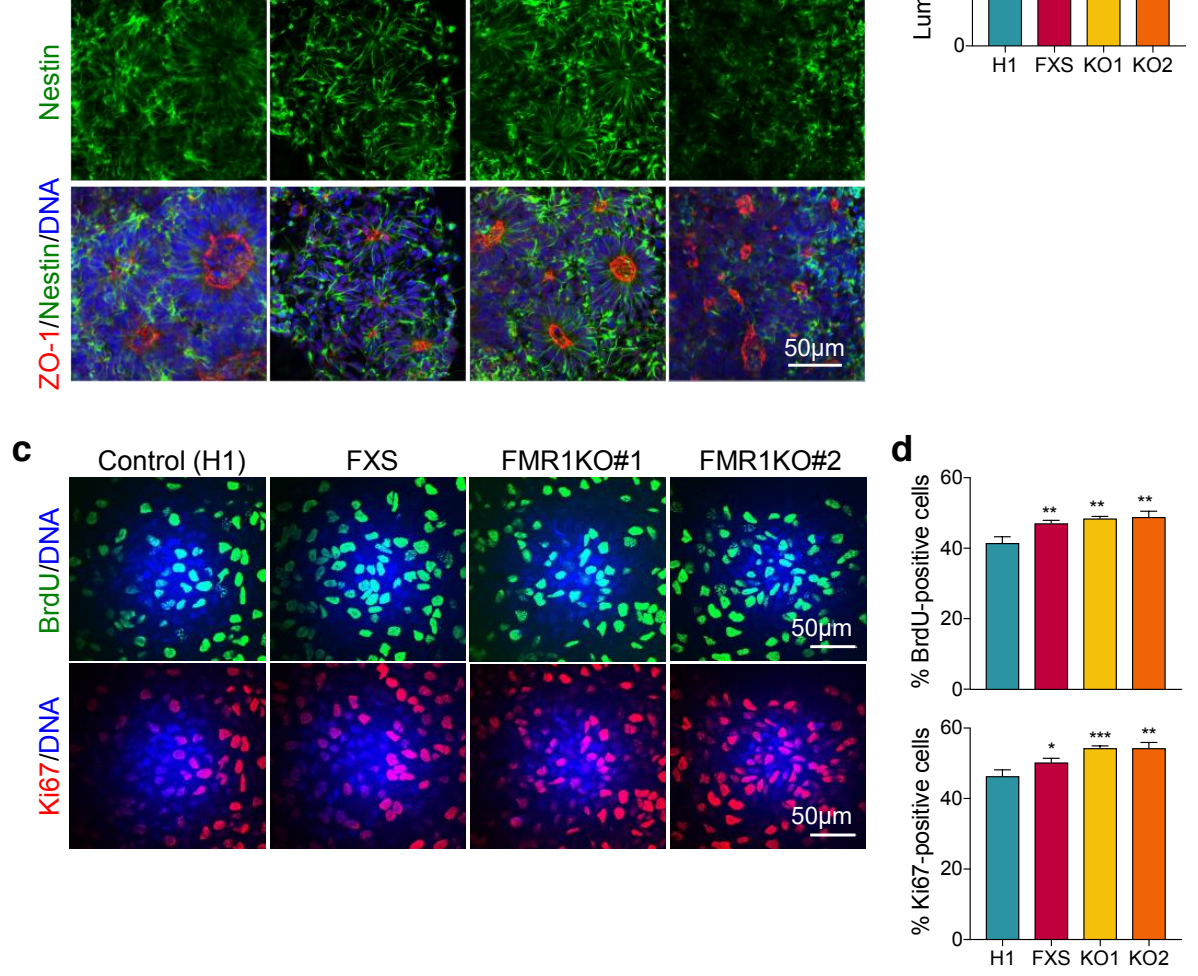

Figure 4. FMRP deficiency leads to abnormal neural rosette formation and increased neural progenitor proliferation. (a) Immunostaining shows neural rosette structures identified using Nestin and ZO-1 expression. (b) Quantification of lumen size area (based on ZO-1 positive staining). Lumen area was measured by ImageJ software. Values shown as mean \pm SEM based on $n=4$ biological replicates per genotype; ${ }^{*} p<0.05$ and ${ }^{* * *} p<0.001$ compared to control $(\mathrm{H} 1)$ was determined by one-way ANOVA with Tukey's post-hoc test. (c,d) BrdU-labeling and Ki67 reveals increased proliferation in FXS and FMR1KO (KO1) neural progenitor cells compared with control $(\mathrm{H} 1)$. Values shown as mean \pm SEM based on blinded counting of 10 images from each of three biological replicates per genotype; ${ }^{*} p<0.05,{ }^{* *} p<0.01$, and ${ }^{* * *} p<0.001$ compared with control (H1) was determined by one-way ANOVA with Fisher LSD post-hoc test.

body method [37]. Neural rosettes appeared markedly smaller in the FXS and FMR1KO lines compared with the control line (Figure 4a). To quantify the size differences, we measured the area stained with ZO-1, a luminal neural rosette marker, and found it to be significantly reduced in FMR1KO and FXS lines compared with control (Figure $4 \mathbf{b}$ ). Functional annotation of the upregulated genes in FMRP-deficient neurons showed enrichment of transcripts involved in mitosis and cell cycle-related processes. This prompted us to investigate whether cell proliferation is affected in FMRP-deficient cells. Labeling with BrdU and Ki67, two markers of cell proliferation, showed increased FXS and FMR1KO neural progenitor proliferation compared with control cells (Figure $4 \mathbf{c}, \mathbf{d}$ ).

\section{FMRP-deficient neurons exhibit neurite outgrowth deficits and abnormal network connectivity}

Neurite outgrowth is an early neurodevelopmental process critical to the proper formation of axons and dendrites. Neurite outgrowth has been shown to be compromised in a number of intellectual disability and autism disorders, including FXS $[22,24,38]$. To evaluate this 
bioRxiv preprint doi: https://doi.org/10.1101/606038; this version posted April 15, 2019. The copyright holder for this preprint (which was not certified by peer review) is the author/funder, who has granted bioRxiv a license to display the preprint in perpetuity. It is made available under aCC-BY-NC-ND 4.0 International license.

a

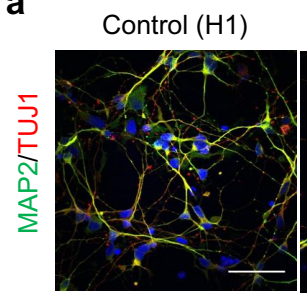

C

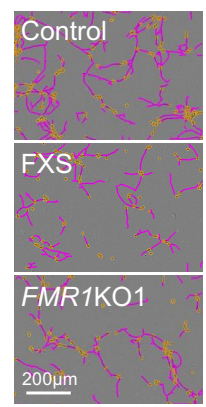

FXS

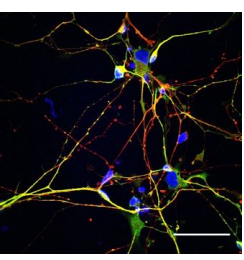

FMR1KO1

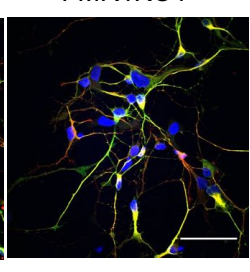

b

d $\rightarrow$ Control $(\mathrm{H} 1) \rightarrow$ FXS $\rightarrow$ FMR1KO
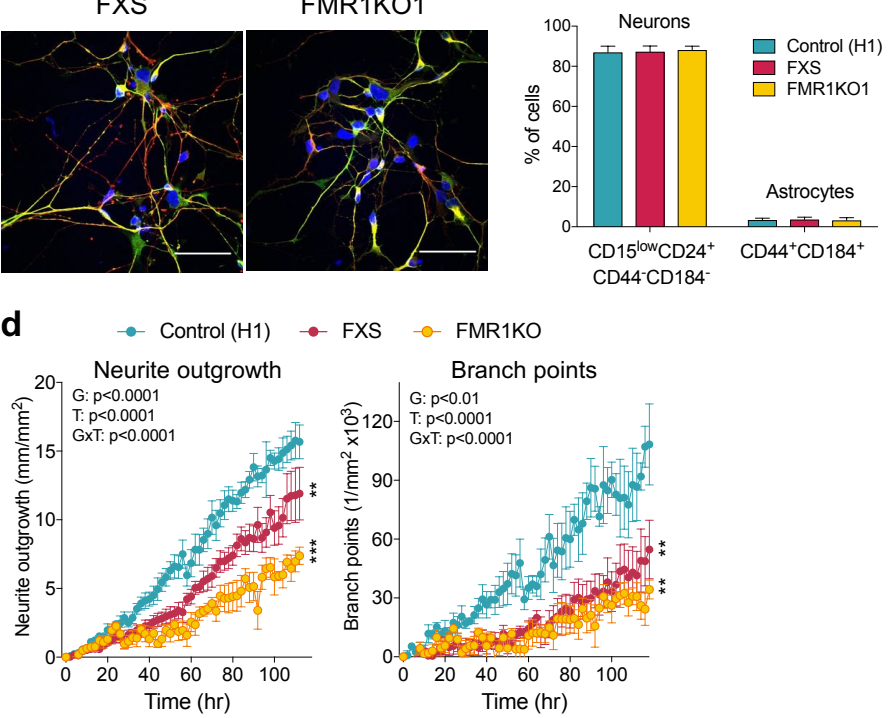

$\mathbf{e}$

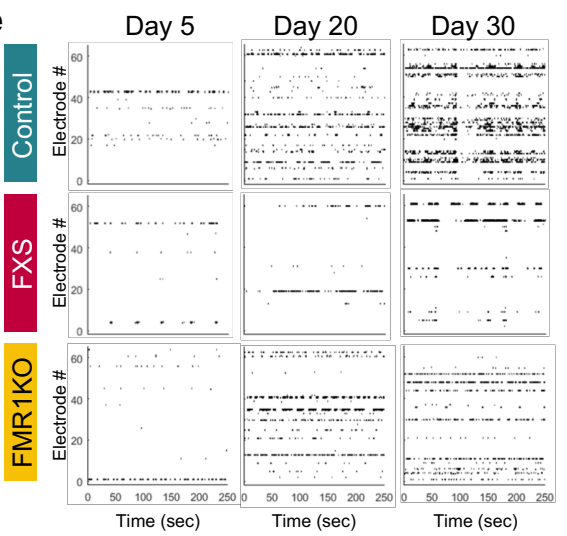

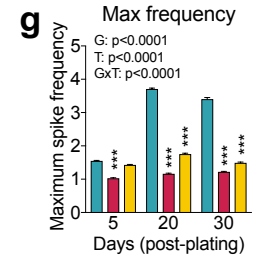

h
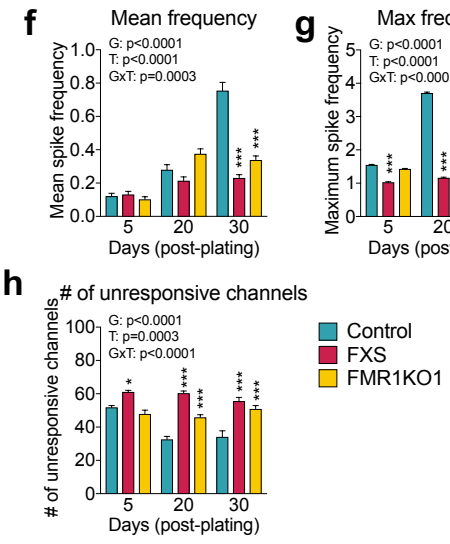

i
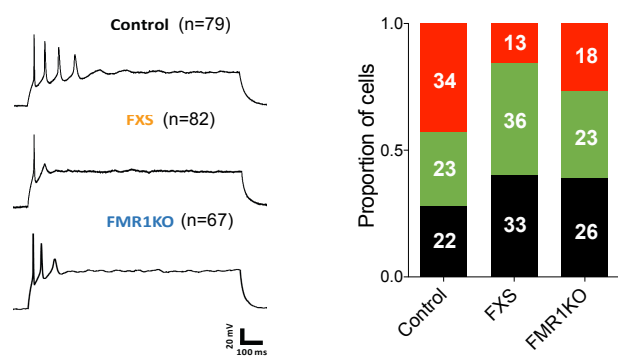

$$
\begin{aligned}
& \text { Multiple } \\
& \text { Single } \\
& \text { None }
\end{aligned}
$$

Figure 5. FMRP-deficient neurons exhibit shorter neurite length and aberrant network connectivity. (a) Immunostaining showing the expression of post-mitotic neuronal markers MAP2 and TUJ1 after 37-40 days of differentiation from hESC; scale-bar = 50 $\mu \mathrm{m}$. (b) Flow cytometry analysis of cellular composition following hPSC neuronal differentiation using cell surface markers of neurons and glia. (c) Representative images of neurite phenotype segmentation. Neurites are labeled in yellow, and the neuronal cell bodies are labeled in pink/purple. (d) Neurite outgrowth and branching measurements. Values shown as mean \pm SD from 6 biological replicates. (e-f) Analysis of neuronal network activity using multi-electrode array (MEA) recordings. $\mathrm{n}=6$ per genotype. (e) Raster plots of spike time stamps indicating neuronal spontaneous activity as measured by MEA recordings. (f) Mean firing (spike) frequency. (g) Maximum firing (spike) frequency. (h) Number of unresponsive channels; ${ }^{*} p<0.01$, ${ }^{* *} p<0.01$, and ${ }^{* * *} p$ $<0.001$ compared to control $(\mathrm{H} 1)$ was determined by two-way ANOVA with Tukey's post-hoc test. G, genotype: T, time. (i) Analysis of intrinsic electrophysiological neuronal properties by patch clamp.

deficit in FMR1KO lines, we performed longitudinal tracking of neurite elongation in hESCderived neurons using live-cell imaging. We found a striking reduction in neurite outgrowth and branching in FMR1KO and FXS neurons over time compared with control neurons (Figure $5 \mathbf{c}, \mathbf{d}$ ). These results demonstrate that isogenic FMR1KO neurons recapitulate this 
FXS-linked morphological deficit.

\section{FMRP-deficient neurons show abnormal electrophysiological network connectivity}

The reduced neurite outgrowth in FMRP-deficient neurons led us to investigate electrophysiological neuronal network connectivity. We evaluated extracellular spontaneous activity using longitudinal multi-electrode array (MEA) recordings, which enable detection of action potentials (spikes) based on changes in field potential [39]. MEA recordings were performed over a 30-day period starting at day 37 of neuronal differentiation. Representative raster plots depicting neuronal firings (spikes) over a 250 -sec period of continuous recording on days 5, 20, and 30 are shown in Figure 5e. Spontaneous action potentials were detected for all groups, confirming successful derivation of functional neurons from all three genotypes. However, analysis of spike frequencies revealed profound deficits in the mean and maximum spike frequencies for FMR1KO and FXS neurons compared with control neurons (Figure 5f-h), suggesting altered neuronal network connectivity as a result of FMRP deficiency. As differences in MEA activity can also reflect intrinsic neuronal differences, we analyzed the basic properties of action potential (AP) firing in FMRP-deficient and control neurons using patch-clamp recordings (Figure 5i and Table S4). While there were no differences in the proportion of neurons with a single or no AP between FXS and FMR1KO (69 of 82 for FXS and 49 of 67 for FMR1KO, p > 0.05, Chi2 test), the proportion of such neurons was significantly larger when compared with control neurons (45 of 79 for control, $\mathrm{p}<0.001$ compared with FXS, $\mathrm{p}<0.05$ compared with FMR1KO, Chi2 test). These results suggest that differences in the intrinsic properties of FMRP-deficient neurons may also contribute to the deficits in MEA activity observed.

\section{Discussion}

Isogenic models represent well-controlled experimental systems in which cellular and molecular abnormalities arising from genetic disorders can be readily attributed to the genetic lesion under study [40]. Here, we describe the generation and characterization of isogenic FMR1KO human embryonic stem cells as a model of FXS. We illustrate alterations of several biological pathways important to brain development and function in FMRPdeficient neurons at both the RNA and protein level. We functionally validate alterations in a number of these pathways, showing phenotypic deficits including abnormal neural rosette formation and increased neural progenitor cell proliferation. We further demonstrate neurite outgrowth and branching deficits in FMRP-deficient neurons along with impaired electrophysiological neuronal connectivity. Our findings reveal key molecular signatures and neurodevelopmental abnormalities arising from loss of FMRP.

We find that neurons lacking FMRP show dysregulation of genes and proteins related to diverse cellular and neuronal processes. It is interesting to note that there was a greater number of differentially expressed genes and proteins between the isogenic lines (FMR1KO versus control) compared with the non-isogenic ones (FXS versus control). The use of isogenic lines minimizes the potential for not only false positives but also false negatives, and it is possible that a large number of false negatives in the FXS versus control comparison due to differences in the genetic background resulted in the smaller number of differentially expressed genes and proteins in that group.

The cellular and neuronal processes dysregulated in FMRP-deficient neurons include nervous system development, neuronal differentiation, cell cycle progression, as well as 
neurotransmission. We were able to corroborate a number of these changes functionally. Firstly, we discovered that the formation of neural rosettes is abnormal in the absence of FMRP. Neural rosettes are self-assembling structures considered surrogates of early neurulation and neural tube formation [41,42]. Secondly, a number of cell cycle genes and proteins were found to be dysregulated in FXS and FMR1KO neurons in our transcriptome and proteome analysis. These include, among others, DNA replication initiators and regulators of cell cycle checkpoints. Cell cycle analysis, using neural progenitor proliferation as an assay, showed increased proliferation of FXS and FMR1KO neural progenitors compared with control cells. In agreement with these results, studies using murine and fly models of FXS have shown that FMRP functions in the regulation of timing and proliferative capacities of neural progenitors, with loss of FMRP leading to increased neural progenitor proliferation [43-45]. These findings suggest altered cell cycle dynamics in FXS.

A neurodevelopmental feature important for neuronal connectivity is the branching and extension of neurites that develop into axons and dendrites, which precede the formation of synaptic connections [24]. Our observation of profound deficits in neurite outgrowth and branching in FMR1KO and FXS neurons supports previous reports showing reduced neurite development in FMRP-deficient mouse primary neurons $[43,46]$ as well as hESCand hiPSC-derived neurons [20-22]. One exception is a study examining FXS neurons derived from human fetal cortical neurospheres where no difference in neurite length or branching between FXS and control neurons was found [47]. This discrepancy may be due to the small sample size ( $n=1$ FXS and $n=2$ control post-mortem fetal cortices) and the non-isogenic settings employed.

The mechanisms underlying abnormal neurite growth phenotype have not been investigated to date but are likely to involve dysregulation of one or more developmental pathways related to axon guidance and extension. This notion is supported by our RNA-seq and proteome analysis, where we find enrichment of dysregulated genes related to axon guidance, neurite outgrowth, and cell adhesion, such as CNTN2, ANK3, GAP43, KIF5A, EFNB3, CNTN4, UNC5A, NTNG1, DSCAM, ROBO2, PTPRT, CNTNAP2, GPRIN3, KIF5C and CNTN1. As an example, CNTN4 encodes Contactin-4, which is an axon-associated cell adhesion molecule that functions in neuronal network formation and plasticity [48]. DSCAM encodes Down Syndrome Cell Adhesion molecule that plays a role in neuronal self-avoidance [49]. ROBO2 encodes for Roundabout, Axon Guidance Receptor, Homolog 2 that promotes axon guidance and cell migration. PTPRT, an FMRP substrate [50], is a protein tyrosine phosphatase, receptor Type $\mathrm{T}$ involved in synapse formation and dendritic arborization [50]. NTNG1, encoding for Netrin-G1 receptor, is involved in promoting neurite outgrowth of axons and dendrites [51]. CNTNAP2, an autism-linked gene encoding a neurexin-related cell-adhesion molecule, has been shown to influence neurite outgrowth $[52,53]$. Downregulation of these key genes and associated proteins might account for the neurite outgrowth phenotype in FMRP-deficient neurons. Interestingly, some of these genes (DSCAM, NTNG1, UNC5A, GAP43, CNTN4, CNTNAP2, GPRIN3, KIF5C) have been associated with other neurodevelopmental disorders [54-58].

One potential consequence of neurite growth deficits is reduced neuronal network connectivity. Indeed, the significant reduction in spontaneous firings we observed using longitudinal MEA recordings in FMRP-deficient neurons is consistent with this notion. On the other hand, patch clamp recordings of neurons derived from FXS hESCs have also shown impaired ability to fire repetitive action potentials, and had reduced inward/outward currents [24]. This is consistent with our patch clamping recordings where we find a significantly reduced proportion of FMRP-deficient neurons that fire multiple action potentials compared with control. Furthermore, a well-known role for FMRP is in the regulation of 
key proteins involved in synaptic function and neurotransmission [59]. This is corroborated by our transcriptome and proteome analyses where we observed dysregulation of genes involved in neuronal excitability, neurotransmitter secretion, and synaptic transmission such as potassium channels in FMRP-deficient neurons. Thus, the reduced spontaneous firings we observe in FXS and FMR1KO neurons are likely the consequence of both impaired neuronal connectivity as well as altered intrinsic firing properties.

Our transcriptome and proteome analyses delineate dysregulated pathways affected in the absence of FMRP, highlighting mechanisms of potential relevance to the clinical manifestations of FXS. Overall, our study describes an isogenic hPSC-based model of FXS that can serve as a platform to investigate the pathophysiology of disease, and to screen and validate targets of therapeutic potential, in the context of human neurons.

\section{Materials and Methods}

Cell culture. HEK293 cells for assessing CRISPR/Cas9 activity were cultured in Dulbecco's Modified Eagle's Medium (DMEM), supplemented with 10\% Fetal Bovine Serum (FBS). Control H1 hESCs (WiCell, Wisconsin) and WCMC-37 FXS hESCs (obtained from Nikica Zaninovic, Weill Cornell Medical College of Cornell University, New York) were grown using feeder-free conditions and passaged on Matrigel (BD Biosciences, Franklin Lakes, NJ)coated plates in mTESR1 medium (STEMCELL Technologies, Vancouver, Canada). Cells were passaged by 7-min incubation with Dispase (STEMCELL Technologies, Vancouver, Canada). All hESCs have tested negative for mycoplasma.

Generation of isogenic FMR1 knockout (KO) lines. To create FMR1 KO lines, CRISPR/ Cas9 was used to target the third exon of FMR1 using a gRNA with the following sequence: 5-TTTACAGCTGGCAGCCTGATAGG-3. gRNA was cloned into pSpCas9(BB)-2A-Puro (PX459) V2.0 (Addgene plasmid \# 62988), which contains a puromycin cassette to facilitate selection of transfected cells. The cloned vector was electroporated into H1 hES cells using the Neon Transfection System (Thermo Fisher) at 1,400 V for 3 pulses of $10 \mathrm{~ms}$. Puromycin $(1 \mu \mathrm{g} / \mathrm{ml})$ was added to the culture $48 \mathrm{~h}$ after electroporation for a period of $48 \mathrm{~h}$ to enrich for transfected cells. Colonies derived from puromycin-resistant cells were picked and expanded for screening. PCR amplification using the following primers 5-TGCTTGGGAATTAGAGGGCA-3 (forward) and 5-TTGCGGCAGTGACTTTCAAA-3 (reverse), followed by the Surveyor Assay (following the manufacturer's instructions), were used to identify correctly targeted clones. Two clones, harboring frameshift indels in FMR1, were selected for further characterization.

Karyotyping. Karyotypes were determined from G-banding analysis using standard protocol according to the ISCN nomenclature. Karyotyping was performed as a service by the DNA Diagnostic and Research Laboratory at the KK Women and Children's Hospital, Singapore.

Testing for genomic Cas9 integration. To assay for possible genomic integration of Cas9, 100ng of purified DNA extracted from H1, FXS, KO1, KO2 along with Cas9 donor plasmid were used as template DNA and amplified with F 5'-GAAGAAGAATGGCCTGTTCG-3' and R 5'-GCCTTATCCAGTTCGCTCAG-3' with KOD Xtreme (Novagen, \#71975). Cycling conditions were as follows: initial denaturation at $96^{\circ} \mathrm{C}$ for $5 \mathrm{mins}$, followed by 30 cycles of $96^{\circ} \mathrm{C}$ for $45 \mathrm{~s}, 70^{\circ} \mathrm{C}$ ramped to $58^{\circ} \mathrm{C}\left(-0.2^{\circ} \mathrm{C} / \mathrm{s}\right.$, total duration of $\left.1 \mathrm{~min}\right)$ and $72^{\circ} \mathrm{C}$ for $3 \mathrm{mins}$ 
and a final elongation at $72^{\circ} \mathrm{C}$ for 10 mins. Amplicons were visualized on $1 \%$ agarose gel on the Geldoc XR system (Bio-Rad).

Differentiation of three germ layers. To form embryoid bodies (EBs), hESCs were dissociated into clumps using Dispase and cultured on low-attachment tissue culture plates in KSR medium (DMEM/F12 with 20\% Knock-Out Serum, 1\% GlutaMax, \% non-essential amino acids, and $0.1 \mathrm{mM} 2$-mercaptoethanol). Medium was changed every two days for a total of seven days. EBs were then harvested onto Matrigel-coated coverslips and left to spontaneously differentiate for nine days in KSR medium before fixation with $4 \%$ paraformaldehyde and staining.

Neural differentiation. hESCs were induced into neural progenitor cells (NPCs) according to a previously published protocol [29]. Briefly, single-cell dissociated hESC at a density of 30,000 cells/cm2 were plated in neural induction media (NIM, DMEM/F12:NeuroBasal media $1: 1$ with $1 \%$ N2, 2\% B27, 1\% PenStrep, 1\% GlutaMax, $10 \mathrm{ng} / \mathrm{ml} \mathrm{hLIF}$, and $5 \mu \mathrm{g} / \mathrm{ml}$

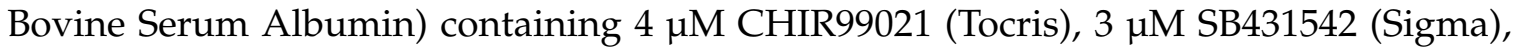
and $0.1 \mu \mathrm{M}$ Compound $\mathrm{E}$ (Millipore) for the first seven days. The culture was then split at a 1:3 ratio for the next five passages using Accutase in NIM without Compound E on Matrigel-coated plates.

For neuronal differentiation, NPCs were plated at a density of 20,000 cells/cm2 on 50 $\mu \mathrm{g} / \mathrm{ml}$ poly-L-ornithine $/ 10 \mu \mathrm{g} / \mathrm{ml}$ laminin-coated plates and grown in NeuroDiff media (DMEM/F12/Neurobasal media (1:1) supplemented with 1\% N2, 2\% B27, $20 \mathrm{ng} / \mathrm{ml}$ GDNF (RD Systems), $20 \mathrm{ng} / \mathrm{ml}$ BDNF (RD Systems), $300 \mu \mathrm{M}$ dibutyryl-cyclic AMP (D0260, Sigma Aldrich), and $200 \mathrm{nM}$ L-Ascorbic Acid (A4403, Sigma Aldrich)) for at least three weeks. Medium was changed every 2-3 days.

Flow cytometry analysis of neurons. Differentiated neurons were analysed by flow cytometry as described previously [30]. Briefly, at day 37 of differentiation, neurons were dissociated with Accutase for 10 minutes, and subsequently washed in DMEM/F12 to remove the Accutase. Dissociated neurons were treated with DNAse I for 10 minutes at room temperature and strained through a $70 \mu \mathrm{m}$ cell strainer. Neurons were washed and resuspended in NeuroDiff sorting medium, consisting of NeuroDiff media with the addition of $0.5 \%$ BSA, $50 \mu \mathrm{g} / \mathrm{ml}$ Gentamycin and $0.5 \mathrm{mM}$ EDTA. Cells were stained with fluorochrome-conjugated antibodies (anti-CD15-V450-\#642922, anti-CD24-PE-Cy7 \#561646, anti-CD44-PE \#550989, anti-CD184-PE-\#555974; BD Biosciences) for 30 minutes on ice. Cells were washed and resuspended in NeuroDiff sorting medium at a concentration of 2 million cells $/ \mathrm{ml}$. Fluorescence activated cell sorting was performed with a FACSAria II (BD Biosciences) with a $100 \mu \mathrm{m}$ nozzle. Neurons were quantified based on the expression of CD184-, CD44-, CD15LOW, CD24+, and non-neuronal cells (glia) were quantified based on the expression of CD44+ and CD184+.

Neurite outgrowth measurements. To assess neurite outgrowth, NPCs were plated at a density of 15,000 cells/cm2 in NeuroDiff media with modifications (substituting N2 with CultureOne supplement (Thermo Fisher)). The plate was imaged using an IncuCyte Zoom Imaging system (Essen Bioscience, Ann Arbor, MI) every $2 \mathrm{~h}$ for five days. For each genotype, quadruplicate live-capture measurements were performed in 9 image field per well. Cells were imaged under phase contrast, and analysis was performed using IncuCyte's NeuroTrack module. The growth rate of neurites in each well was obtained by measuring 
the surface area covered by neurites and expressed as $\mathrm{mm} / \mathrm{mm} 2$. The neurite outgrowth experiments have been repeated more than 3 times.

Immunofluorescence staining. Cells were plated on ethanol-treated coverslips and fixed with $4 \%$ formaldehyde in phosphate buffer saline (PBS) for $15 \mathrm{~min}$ at room temperature. After washing with Tris-Buffered Saline (TBS), cells were incubated in blocking buffer (TBS containing 5\% goat serum, 1\% Bovine Serum Albumin, and 0.1\% Triton-X-100 (Sigma Aldrich)) for $45 \mathrm{~min}$ at room temperature. Primary antibodies were incubated with fixed cells overnight at $4^{\circ} \mathrm{C}$ in blocking buffer without Triton-X-100. The following primary antibodies were used: anti-FMRP mouse monoclonal (MAB2160, Merck-Millipore), anti-OCT-4 rabbit polyclonal (sc-9081, Santa Cruz), anti-SSEA4 mouse monoclonal (MAB4304, MerckMillipore), anti-AFP (ST1673, Merck-Millipore), anti-ASM-1 mouse monoclonal (CBL171, Merck-Millipore), anti-MAP2 rabbit polyclonal (AB5622, EMD Millipore), anti-TUJ1 mouse monoclonal (MAB1637, Merck-Millipore), anti-TBR1 rabbit polyclonal (AB31940, Abcam), anti-GABA rabbit polyclonal (A2052, Sigma), anti-PAX6 rabbit polyclonal (PRB-278P, BioLegend), anti-Nestin mouse monoclonal (MAB5326, Merck-Millipore), anti-ZO-1 rabbit polyclonal (617300, Merck-Millipore), and anti-Ki67 mouse monoclonal (MAB4190, MerckMillipore). Cells were subsequently stained with secondary antibodies conjugated to Alexa Fluor 555 or 488 (Molecular Probes, Thermo Fisher) for $1 \mathrm{~h}$ at room temperature in the dark and incubated with $1 \mu \mathrm{g} / \mathrm{ml}$ 4',6-diamidino-2-phenylindole (DAPI, Sigma Aldrich) for 10 min. Images were captured using an FV1000 Inverted Confocal System.

Immunocytochemistry quantification analysis. To determine the proportion of positive cells, images were captured from at least 10 randomly selected areas. Quantification for each sample/genotype was performed blindly in a minimum of 3 coverslips. ImageJ software was then used to compute the total number of cells (DAPI-stained nuclei) and the number of cells expressing the markers. All data were expressed as the mean and standard error of the mean from three independent replicates. Statistical analysis was carried out using one-way ANOVA with Tukey's post-hoc analysis, and significance was defined with $\mathrm{p}$-value $<0.05$.

Immunoblotting. Cells were lysed with RIPA buffer (Sigma Aldrich) containing cOmplete Protease Inhibitor cocktail tablets (Roche). Protein concentration was measured using the Bradford assay (BioRad). The samples were denatured at $70^{\circ} \mathrm{C}$ for $10 \mathrm{~min}$ in $4 \times$ NuPAGE sample buffer and 10 $\times$ NuPAGE reducing agent (Thermo Fisher). A total of $30 \mu \mathrm{g}$ of protein per sample was separated on 4-12\% Bis-Tris gradient gels in MOPS SDS running buffer (Thermo Fisher) at $100 \mathrm{~V}$ for $3 \mathrm{~h}$ followed by transfer to nitrocellulose membrane at $120 \mathrm{~V}$ for $1.5 \mathrm{~h}$ at room temperature. The following primary antibodies were used for detection: anti-FMRP (MAB2160, Millipore; 6B8, Biolegend), and anti-Calnexin (Sigma, C4731). AlexaFluor 680 goat anti-mouse (Thermo Fisher) and DyLight 800 goat anti-rabbit (Rockland) were used as secondary antibodies. Membranes were imaged using the Li-Cor Odyssey infrared imaging system.

MEA Recordings. Neurons on day 37 of differentiation were dissociated and re-plated on $0.1 \%$ polyethylenimine (Sigma Aldrich)-coated MEA plates (Axion Biosystems) in BrainPhys media (STEMCELL Technologies, Vancouver, Canada) supplemented with BDNF, GDNF, cAMP, and L-Ascorbic Acid as previously described [37,60]. Spontaneous activity was recorded at $37^{\circ} \mathrm{C}$ for 5 min every 2-3 days for 30 days using the Maestro MEA 
System (Axion Biosystem). Neural signals were sampled at $12.5 \mathrm{kHz}$, high-pass filtered $(200 \mathrm{~Hz}-3 \mathrm{kHz})$, and a threshold based on six standard deviation above noise levels was set (Axion Integrated Studio software (AxIS). Detected timestamps were analyzed using custom-written Matlab scripts (R2015b) as described previously [60]. Spike frequency is calculated as [spike count / experiment duration]. Mean spike frequency function removes outliers, then calculates mean \pm SEM excluding channels where no activity was detected (spike number $<=1$ ). $\mathrm{N}$ represents the total number of channels where activity was detected from all wells of that particular condition.

Patch clamp recording. Electrophysiological recordings were conducted on neurons cultured on glass coverslips. The experiments were performed in the blinded sample groups. The coverslips were transferred to a recording chamber in standard recording medium containing the following (in $\mathrm{mM}$ ): $124 \mathrm{NaCl}, 24 \mathrm{NaHCO}$, 13 Glucose, 5 HEPES, 2.5 $\mathrm{KCl}, 2 \mathrm{MgSO} 4,2 \mathrm{CaCl} 2$ and $1.2 \mathrm{NaH} 2 \mathrm{PO} 4$ (310 mOsm, pH 7.4). Cells were patch-clamped with pipettes containing the following (in $\mathrm{mM}$ ): $130 \mathrm{~K}$-Gluconate, $11 \mathrm{KCl}, 10 \mathrm{HEPES}, 5 \mathrm{NaCl}$, 5 Na-phosphocreatine, $2 \mathrm{Mg}$-ATP, $1 \mathrm{MgCl} 2,0.3 \mathrm{Na}-\mathrm{GTP}$ and 0.1 EGTA (pH 7.4, $300 \mathrm{mOsm}$ ). Action potentials are evoked by injecting depolarizing current pulses in current-clamp mode. Signals were amplified with EPC 10 USB (HEKA Elektronik, Germany) and recorded with the accompanying PATCHMASTER software. Data were analyzed using AxoGraph Ver 1.7.0 (AxoGraph Company, CA, USA) and GraphPad Prism Ver 6.00 (GraphPad Software, CA, USA).

RNA isolation, cDNA synthesis, and Quantitative PCR. Cells were lysed using RLT Plus buffer and RNA was purified using the RNeasy Plus Mini kit (Qiagen) according to the manufacturer's instructions. For all samples, cDNA was generated in $20 \mu 1$ reactions using High-Capacity Reverse Transcriptase kit (ABI, Thermo Fisher). Quantitative real time PCR (qRT-PCR) reactions were performed in the StepOnePlus or Quant Studio6 Flex Real Time PCR System (ABI, Thermo Fisher) with ten-fold dilution of cDNA and $200 \mathrm{nM}$ of each primer using the SYBR Select PCR Master Mix (ABI, Thermo Fisher). Primers are listed in Table S1. Relative gene expression levels were calculated using the comparative Ct method, and normalized against a control with human GAPDH.

RNA-seq. RNA was extracted from cells on day 37 of neuronal differentiation using the RNeasy Plus Mini kit (Qiagen) according to the manufacturer's instructions. Subsequent library preparation and paired-end 150-bp sequencing and 20 million reads/per sample using HiSeq4000 were performed by Novogene (Hong Kong). We performed RNA-seq quantification directly from the reads with Salmon v0.9.1 [61] using GRCh38 (hg38; Ensembl release 87) human reference genome. The data was imported into $R$ using tximport [62] and analyzed for differentially expressed genes with DESeq2 [63]. Significant hits were identified using FDR $<0.05$ cut-off and divided into upregulated $(\log 2 \mathrm{FC}>0)$ and downregulated $(\log 2 \mathrm{FC}<0)$ genes. The results were visualized using volcano plots. Statistical analyses for the RNA-seq and proteome data, were performed using R statistical software [64] and Python 3.6 (www.python.org). Functional enrichment analysis of significant hits was performed in clueGO version 2.5.2 [65]. For exploratory analysis, we initially normalized the read counts using the variance stabilizing transformation (VST) algorithm [66] for negative binomial data with a dispersion-mean trend. Sample-to-sample distances were calculated using the dist function [67-69] and plotted as heatmap to assess for the quality of the samples. Principal component analysis (PCA) performed in R showed a good separation of the groups, using only 2 principal components. Genes found significantly regulated in 
both FXS andFMR1KO cells, with the same direction of fold change, were considered of interest and their normalized expression visualized in the form of a heatmap.

Preparations of samples for mass spectrometry analysis. For proteomic mass spectrometry (MS) analysis, cells were harvested on day 37 of neuronal differentiation. Sample preparation was performed as described previously $[70,71]$ with optimization for pellet cells. 100ul of SDC reduction and alkylation buffer were added and the mixture was boiled for $10 \mathrm{~min}$ to denature proteins. After cooling down, the proteolytic enzymes LysC and trypsin were added in a 1:100 $(\mathrm{w} / \mathrm{w})$ ratio. Digestion was performed overnight at $37^{\circ} \mathrm{C}$. Peptides were acidified using tri-fluoro-acetic acid (TFA) to a final concentration of $0.1 \%$ for SDB-RPS binding and 20ug was loaded on two StageTip plugs. The StageTips were washed twice with $1 \%$ TFA and once with $0.2 \%$ TFA and centrifuged at 500xg. After washing the purified peptides were eluted by $60 \mathrm{ul}$ of elution buffer $(80 \%$ acetonitrile, $19 \%$ ddH2O, $1 \%$ ammonia). The collected material was completely dried using a SpeedVac centrifuge at 45C (Eppendorf, Concentrator plus). Peptides were suspended in buffer $A^{*}$ ( $5 \%$ acetonitrile, $0.1 \%$ TFA) and afterwards mixed for 10 minutes at 1000rpm. Peptide concentrations were determined by Nanodrop (Thermo Fisher Scientific) measurement at A280nm and sample concentrations were adjusted to 1.0ug per injection.

LC-MS/MS Analysis. All peptide samples were analyzed with nanoflow Easy-nLC 1200 (Thermo Fisher Scientific, Denmark) coupled to Q Exactive HF-X mass spectrometers (Thermo Fisher Scientific, Denmark). Peptides were separated on in-house packed column (75 m inner diameter $\times 50 \mathrm{~cm}$ length) with $1.9 \mathrm{~m} \mathrm{C18}$ beads (Dr. Maisch, Germany). Column temperature was kept at $60^{\circ} \mathrm{C}$. Peptide separation was achieved by $100 \mathrm{~min}$ gradients. Peptides were loaded with $0.1 \%$ formic acid and eluted with a nonlinear gradient of increasing buffer B ( $0.1 \%$ formic acid and $80 \%$ acetonitrile) and decreasing buffer $\mathrm{A}(0.1 \%$ formic acid) at a flow rate of $350 \mathrm{nl} / \mathrm{min}$. Buffer B was increased slowly from $2 \%$ to $220 \%$ over 55 minutes and ramped to $40 \%$ over 40 minutes and then to $98 \%$, where it was held for 5 minutes before being drop down to $2 \%$ again for column re-equilibration. Q Exactive HF-X mass spectrometer was operated in positive polarity mode with capillary temperature of 275 ${ }^{\circ} \mathrm{C}$. Full MS survey scan resolution was set to 60,000 with an automatic gain control target value (AGC) of $3 \times 10^{6}$ using a scan range of $3501650 \mathrm{~m} / \mathrm{z}$ and maximum injection times (IT) of $15 \mathrm{~ms}$. This was followed by a data-dependent higher-energy collisional dissociation (HCD) based fragmentation (normalized collision energy=28) of up to 15 most abundant precursor ions. The MS/MS scans were obtained at 15,000 resolution with AGC target of $5 \times 10^{4}$ and maximum injection time of $25 \mathrm{~ms}$. Repeated sequencing of peptides was reduced by dynamically excluding previously targeted peptides for 30 seconds.

MaxQuant Data Processing. All data files were analyzed using the MaxQuant software suite 1.5.5.1 (www.maxquant.org) with the Andromeda search engine [72]. MS/MS spectra were searched against an in silico tryptic digest of Homo Sapiens proteins from the UniProt sequence database. All MS/MS spectra were searched with the following MaxQuant parameters for peptide identification: acetyl and methionine oxidation were searched as variable modifications and cysteine carbamidomethylation was set as fixed modification with maximal 2 missed cleavages. Precursors were initially matched to $4.5 \mathrm{ppm}$ tolerance and $20 \mathrm{ppm}$. The false discovery rate (FDR) for protein and peptide matches was set to $1 \%$ based on Andromeda score, peptide length, and individual peptide mass errors. Peptide length was minimum 7 amino acids, minimum Andromeda score was 40 and maximal peptide mass was $4600 \mathrm{Da}$. The second peptide feature was enabled. The match between 
runs option was also enabled with a match time window of $0.7 \mathrm{~min}$ and an alignment time window of $20 \mathrm{~min}$. Relative label-free quantification (LFQ) was done using the MaxLFQ algorithm integrated into MaxQuant [73]. Protein quantification needed minimum two unique or razor peptides per protein group and minimum ratio count was set to 2 .

Proteome. Protein abundance quantitative data generated in MaxQuant was used as input for the downstream statistical analysis. The number of peptides and proteins identified in each experiment was extracted from the data and used to access the quality of the runs. During the preprocessing of the data, protein LFQ intensities were normalized by log2 transformation. Furthermore, potential contaminants and proteins identified in the decoy reverse database or only by site modification were excluded, resulting in a total of 5007 protein groups. In order to reduce the noise in the data, we filtered out proteins that did not fulfill the requirement of three valid values in at least one group. The missing values were then imputed with values randomly drawn from a downshifted Gaussian distribution of each sample's valid values (downshift $=1.8$ standard deviations, width $=0.3$ standard deviations) [74]. To examine the correlation between experiments, we calculated Pearson's correlation of the protein groups intensities and generated a heatmap. PCA was performed and visualized with 2 principal components, showing clear separation between the three groups. Differentially expressed proteins in FXS and FMR1KO cells were identified by Student's t-test with Benjamini-Hochberg FDR correction for multiple hypotheses. The results were visualized with volcano plots and a cut-off of FDR $<0.05$ was used to select significant hits, further divided into significant upregulated $(\log 2 \mathrm{FC}>0)$ and significant downregulated $(\log 2 \mathrm{FC}<0)$ hits. Hierarchical clustering of significant hits was carried out on the z-score protein intensities using Euclidean distance.

Enrichment analysis and annotation networks. Functional enrichment analysis of significant hits was performed in was performed in Cytoscape 3.6 [75] clueGO app (version 2.5.2) [65], applying Fischer's exact test and Benjamini-Hochberg FDR correction and using GOBP, GOCC, GOMF, and Reactome annotations. All genes identified in RNA-seq experiments and proteins selected for missing value imputation in Proteomics experiments, were used as background. A cut-off of FDR $<0.05$ was used to identify all significantly enriched terms.

To facilitate the visualization of enriched terms in a network, we applied hierarchical level cut-offs to the enrichment search, as well as a minimum number of genes of interest per term. To reduce the redundancy of GO terms, the fusion option was also selected. GO terms, represented as nodes with sizes reflecting their statistical significance, are grouped and linked based on the similarity of their associated genes (kappa score 0.35), with the most significant term per group shown in bold and larger font.

A separate enrichment analysis for significant genes and proteins was performed against the SFARI dataset (downloaded from gene.sfari.org on 07/09/2018) [32] and FMRP targets dataset [4], using Chi2 test. An additional enrichment analysis for the genes found significantly regulated in both RNA-seq and Proteomics was also performed. In this case, common overall genes between the two data types were used as background.

StringDB networks. Significantly regulated genes in both RNA-seq and Proteomics experiments were analyzed, using STRING database [76], to find meaningful protein-protein relationships. The resulting networks were plotted using the cluster_edge_betweenness function [77] to identify highly connected nodes, while the nodes were colored according to their average fold change. 
Neural rosette formation assay. hESCs were dissociated into single cells using Accutase and $4.5 \times 10^{6}$ cells were seeded into AggreWell800 plates (STEMCELL Technologies, Vancouver, Canada) to form neural aggregates in STEMdiff Neural Induction Medium (STEMCELL Technologies, Vancouver, Canada). On day 5, neural aggregates were harvested and transferred into poly-L-ornithine/laminin-coated plates. On day 10, cells were fixed using 4\% paraformaldehyde and stained with antibodies against ZO-1 (rabbit polyclonal, \#617300, Merck-Millipore) and Nestin (mouse monoclonal, MAB5326, Merck-Millipore).

Cell proliferation assay. Approximately $70 \%$ confluent neural progenitor cells were treated with $50 \mu \mathrm{M}$ BrdU for $6 \mathrm{~h}$, followed by fixation with $4 \%$ formaldehyde for $15 \mathrm{~min}$ at room temperature. For antigen retrieval, coverslips were incubated serially three times in ice-cold $1 \mathrm{~N} \mathrm{HCl}$ for $10 \mathrm{~min}, 2 \mathrm{~N} \mathrm{HCl}$ for $10 \mathrm{~min}$ at room temperature, $2 \mathrm{~N} \mathrm{HCl}$ for $20 \mathrm{~min}$ at $37^{\circ} \mathrm{C}$, and lastly in $1 \mathrm{M}$ borate buffer for $10 \mathrm{~min}$. Immunofluorescence staining and imaging was performed as described in the Immunofluorescence staining section using anti-BrdU (sc56258, Santa Cruz) and anti-Ki67 (MAB4190, Millipore) antibodies.

Statistical analysis for biological assays. Samples were processed blind for assays, e.g. immunofluorescence quantitative measurements and electrophysiology. The Shapiro-Wilk test was used to test for normality. Details of the statistical tests used for the different analyses is reported in the respective figure legends. Statistical analysis was carried out GraphPad Prism v7 (La Jolla, CA, USA).

\section{Conflict of interest}

The authors declare no conflict of interest.

\section{Acknowledgments}

We thank members of the Pouladi lab for helpful discussions and comments, members of the NNF-CPR Mass Spectrometry Platform for instrument support and technical assistance, and Dr. Nikica Zaninovic (Weill Cornell Medical College) for the WCMC-37 FXS hESC line. The work was partly funded by a Strategic Positioning Fund for Genetic Orphan Diseases (SPF2012/005) and SUREKids (IAF311019) from the Agency for Science Technology and Research (Singapore) to M.A.P. and a FRAXA Fellowship to K.H.U, and in part supported by a Joint Council Office grant (BMSI/15-800003-SBIC-00E) and the Novo Nordisk Foundation Center for Protein Research.

\section{References}

[1] R. J. Hagerman, E. Berry-Kravis, H. C. Hazlett, D. B. Bailey, H. Moine, R. F. Kooy, F. Tassone, I. Gantois, N. Sonenberg, J.-L. Mandel, and P. J. Hagerman, "Fragile X syndrome.," Nature Reviews Disease Primers, vol. 3, p. 17065, Sept. 2017.

[2] C. Bagni, F. Tassone, G. Neri, and R. Hagerman, "Fragile X syndrome: causes, diagnosis, mechanisms, and therapeutics.," The Journal of clinical investigation, vol. 122, pp. 4314-4322, Dec. 2012.

[3] A. J. Verkerk, M. Pieretti, J. S. Sutcliffe, Y. H. Fu, D. P. Kuhl, A. Pizzuti, O. Reiner, S. Richards, M. F. Victoria, and F. P. Zhang, "Identification of a gene (FMR-1) containing a CGG repeat coincident with a breakpoint cluster region exhibiting length variation in fragile X syndrome.," Cell, vol. 65, pp. 905-914, May 1991. 
[4] J. C. Darnell, S. J. Van Driesche, C. Zhang, K. Y. S. Hung, A. Mele, C. E. Fraser, E. F. Stone, C. Chen, J. J. Fak, S. W. Chi, D. D. Licatalosi, J. D. Richter, and R. B. Darnell, "FMRP stalls ribosomal translocation on mRNAs linked to synaptic function and autism.," Cell, vol. 146, pp. 247-261, July 2011.

[5] J. C. Darnell, K. B. Jensen, P. Jin, V. Brown, S. T. Warren, and R. B. Darnell, "Fragile X mental retardation protein targets G quartet mRNAs important for neuronal function.," Cell, vol. 107, pp. 489-499, Nov. 2001.

[6] E. M. Berry-Kravis, L. Lindemann, A. E. Jønch, G. Apostol, M. F. Bear, R. L. Carpenter, J. N. Crawley, A. Curie, V. des Portes, F. Hossain, F. Gasparini, B. Gomez Mancilla, D. Hessl, E. Loth, S. H. Scharf, P. P. Wang, F. Von Raison, R. Hagerman, W. Spooren, and S. Jacquemont, “Drug development for neurodevelopmental disorders: lessons learned from fragile X syndrome.," Nature Reviews Drug Discovery, vol. 92, p. 234, Dec. 2017.

[7] K. De Boulle, A. J. Verkerk, E. Reyniers, L. Vits, J. Hendrickx, B. Van Roy, F. Van den Bos, E. de Graaff, B. A. Oostra, and P. J. Willems, "A point mutation in the FMR-1 gene associated with fragile X mental retardation.," Nature Genetics, vol. 3, pp. 31-35, Jan. 1993.

[8] J. A. Suhl and S. T. Warren, "Single-Nucleotide Mutations in FMR1 Reveal Novel Functions and Regulatory Mechanisms of the Fragile X Syndrome Protein FMRP.," Journal of experimental neuroscience, vol. 9, no. Suppl 2, pp. 35-41, 2015.

[9] L. K. Myrick, M. Nakamoto-Kinoshita, N. M. Lindor, S. Kirmani, X. Cheng, and S. T. Warren, “Fragile X syndrome due to a missense mutation.," European Journal of Human Genetics, vol. 22, pp. 1185-1189, Oct. 2014.

[10] T. M. Kazdoba, P. T. Leach, J. L. Silverman, and J. N. Crawley, “Modeling fragile X syndrome in the Fmr1 knockout mouse.," Intractable E Rare Diseases Research, vol. 3, pp. 118-133, Nov. 2014.

[11] J. K. Davis and K. Broadie, "Multifarious Functions of the Fragile X Mental Retardation Protein.," Trends in genetics : TIG, vol. 33, pp. 703-714, Oct. 2017.

[12] T. A. Comery, J. B. Harris, P. J. Willems, B. A. Oostra, S. A. Irwin, I. J. Weiler, and W. T. Greenough, "Abnormal dendritic spines in fragile X knockout mice: Maturation and pruning deficits," Proceedings of the National Academy of Sciences of the United States of America, vol. 94, pp. 5401-5404, May 1997.

[13] A. L. Bhakar, G. Dölen, and M. F. Bear, "The pathophysiology of fragile X (and what it teaches us about synapses).," Annual review of neuroscience, vol. 35, no. 1, pp. 417-443, 2012.

[14] A. Contractor, V. A. Klyachko, and C. Portera-Cailliau, "Altered Neuronal and Circuit Excitability in Fragile X Syndrome.," Neuron, vol. 87, pp. 699-715, Aug. 2015.

[15] S. J. Chamberlain, "Disease modelling using human iPSCs.," Human molecular genetics, vol. 25, pp. R173R181, Oct. 2016.

[16] M. C. N. Marchetto, C. Carromeu, A. Acab, D. Yu, G. W. Yeo, Y. Mu, G. Chen, F. H. Gage, and A. R. Muotri, "A model for neural development and treatment of Rett syndrome using human induced pluripotent stem cells.," Cell, vol. 143, pp. 527-539, Nov. 2010.

[17] H.-Q. Huo, Z.-Y. Qu, F. Yuan, L. Ma, L. Yao, M. Xu, Y. Hu, J. Ji, A. Bhattacharyya, S.-C. Zhang, and Y. Liu, "Modeling Down Syndrome with Patient iPSCs Reveals Cellular and Migration Deficits of GABAergic Neurons.," Stem Cell Reports, vol. 10, pp. 1251-1266, Apr. 2018.

[18] S. J. Chamberlain, P.-F. Chen, K. Y. Ng, F. Bourgois-Rocha, F. Lemtiri-Chlieh, E. S. Levine, and M. Lalande, "Induced pluripotent stem cell models of the genomic imprinting disorders Angelman and Prader-Willi syndromes.," PNAS, vol. 107, pp. 17668-17673, Oct. 2010.

[19] Y. Tian, I. Voineagu, S. P. Paşca, H. Won, V. Chandran, S. Horvath, R. E. Dolmetsch, and D. H. Geschwind, "Alteration in basal and depolarization induced transcriptional network in iPSC derived neurons from Timothy syndrome.," Genome medicine, vol. 6, no. 10, p. 75, 2014.

[20] S. D. Sheridan, K. M. Theriault, S. A. Reis, F. Zhou, J. M. Madison, L. Daheron, J. F. Loring, and S. J. Haggarty, "Epigenetic characterization of the FMR1 gene and aberrant neurodevelopment in human induced pluripotent stem cell models of fragile X syndrome.," PLoS ONE, vol. 6, no. 10, p. e26203, 2011.

[21] M. Telias, M. Segal, and D. Ben-Yosef, "Neural differentiation of Fragile X human Embryonic Stem Cells reveals abnormal patterns of development despite successful neurogenesis.," Developmental biology, vol. 374, pp. 32-45, Feb. 2013.

[22] M. E. Doers, M. T. Musser, R. Nichol, E. R. Berndt, M. Baker, T. M. Gomez, S.-C. Zhang, L. Abbeduto, and A. Bhattacharyya, "iPSC-derived forebrain neurons from FXS individuals show defects in initial neurite outgrowth.," Stem cells and development, vol. 23, pp. 1777-1787, Aug. 2014. 
[23] T. Halevy, C. Czech, and N. Benvenisty, "Molecular mechanisms regulating the defects in fragile X syndrome neurons derived from human pluripotent stem cells.," Stem Cell Reports, vol. 4, pp. 37-46, Jan. 2015.

[24] M. Telias, L. Kuznitsov-Yanovsky, M. Segal, and D. Ben-Yosef, "Functional Deficiencies in Fragile X Neurons Derived from Human Embryonic Stem Cells.," The Journal of neuroscience : the official journal of the Society for Neuroscience, vol. 35, pp. 15295-15306, Nov. 2015.

[25] M. Telias, Y. Mayshar, A. Amit, and D. Ben-Yosef, "Molecular mechanisms regulating impaired neurogenesis of fragile $X$ syndrome human embryonic stem cells.," Stem cells and development, vol. 24, pp. scd.2015.0220-2365, Sept. 2015.

[26] Y. Zhou, D. Kumari, N. Sciascia, and K. Usdin, “CGG-repeat dynamics and FMR1 gene silencing in fragile X syndrome stem cells and stem cell-derived neurons.," Molecular autism, vol. 7, no. 1, p. 42, 2016.

[27] J. Gerhardt, M. J. Tomishima, N. Zaninovic, D. Colak, Z. Yan, Q. Zhan, Z. Rosenwaks, S. R. Jaffrey, and C. L. Schildkraut, "The DNA replication program is altered at the FMR1 locus in fragile X embryonic stem cells.," Molecular cell, vol. 53, pp. 19-31, Jan. 2014.

[28] D. Colak, N. Zaninovic, M. S. Cohen, Z. Rosenwaks, W.-Y. Yang, J. Gerhardt, M. D. Disney, and S. R. Jaffrey, "Promoter-bound trinucleotide repeat mRNA drives epigenetic silencing in fragile X syndrome.," Science (New York, NY), vol. 343, pp. 1002-1005, Feb. 2014.

[29] W. Li, W. Sun, Y. Zhang, W. Wei, R. Ambasudhan, P. Xia, M. Talantova, T. Lin, J. Kim, X. Wang, W. R. Kim, S. A. Lipton, K. Zhang, and S. Ding, "Rapid induction and long-term self-renewal of primitive neural precursors from human embryonic stem cells by small molecule inhibitors.," PNAS, vol. 108, pp. 8299-8304, May 2011.

[30] S. H. Yuan, J. Martin, J. Elia, J. Flippin, R. I. Paramban, M. P. Hefferan, J. G. Vidal, Y. Mu, R. L. Killian, M. A. Israel, N. Emre, S. Marsala, M. Marsala, F. H. Gage, L. S. B. Goldstein, and C. T. Carson, “Cell-surface marker signatures for the isolation of neural stem cells, glia and neurons derived from human pluripotent stem cells.," PLoS ONE, vol. 6, no. 3, p. e17540, 2011.

[31] M. J. Boland, K. L. Nazor, H. T. Tran, A. Szücs, C. L. Lynch, R. Paredes, F. Tassone, P. P. Sanna, R. J. Hagerman, and J. F. Loring, "Molecular analyses of neurogenic defects in a human pluripotent stem cell model of fragile X syndrome.," Brain : a journal of neurology, vol. 140, pp. 582-598, Mar. 2017.

[32] B. S. Abrahams, D. E. Arking, D. B. Campbell, H. C. Mefford, E. M. Morrow, L. A. Weiss, I. Menashe, T. Wadkins, S. Banerjee-Basu, and A. Packer, "SFARI Gene 2.0: a community-driven knowledgebase for the autism spectrum disorders (ASDs)," Molecular autism, vol. 4, no. 1, p. 36, 2013.

[33] V. Zerbi, G. D. Ielacqua, M. Markicevic, M. G. Haberl, M. H. Ellisman, A. A-Bhaskaran, A. Frick, M. Rudin, and N. Wenderoth, "Dysfunctional Autism Risk Genes Cause Circuit-Specific Connectivity Deficits With Distinct Developmental Trajectories.," Cerebral cortex (New York, NY : 1991), vol. 28, pp. 2495-2506, July 2018.

[34] A. C. Brumback, I. T. Ellwood, C. Kjaerby, J. Iafrati, S. Robinson, A. T. Lee, T. Patel, S. Nagaraj, F. Davatolhagh, and V. S. Sohal, "Identifying specific prefrontal neurons that contribute to autism-associated abnormalities in physiology and social behavior.," Molecular Psychiatry, vol. 61, p. 1, Nov. 2017.

[35] M. H. Willemsen, W. Ba, W. M. Wissink-Lindhout, A. P. M. de Brouwer, S. A. Haas, M. Bienek, H. Hu, L. E. L. M. Vissers, H. van Bokhoven, V. Kalscheuer, N. Nadif Kasri, and T. Kleefstra, "Involvement of the kinesin family members KIF4A and KIF5C in intellectual disability and synaptic function.," Journal of medical genetics, vol. 51, pp. 487-494, July 2014.

[36] R. Dunn, F. Dudbridge, and C. M. Sanderson, "The use of edge-betweenness clustering to investigate biological function in protein interaction networks.," BMC Bioinformatics, vol. 6, p. 39, Mar. 2005.

[37] X. Xu, Y. Tay, B. Sim, S.-I. Yoon, Y. Huang, J. Ooi, K. H. Utami, A. Ziaei, B. Ng, C. Radulescu, D. Low, A. Y. J. Ng, M. Loh, B. Venkatesh, F. Ginhoux, G. J. Augustine, and M. A. Pouladi, “Reversal of Phenotypic Abnormalities by CRISPR/Cas9-Mediated Gene Correction in Huntington Disease Patient-Derived Induced Pluripotent Stem Cells," Stem Cell Reports, vol. 8, pp. 619-633, Mar. 2017.

[38] A. Kathuria, P. Nowosiad, R. Jagasia, S. Aigner, R. D. Taylor, L. C. Andreae, N. J. F. Gatford, W. Lucchesi, D. P. Srivastava, and J. Price, "Stem cell-derived neurons from autistic individuals with SHANK3 mutation show morphogenetic abnormalities during early development.," Molecular Psychiatry, vol. 23, pp. 735-746, Mar. 2018.

[39] M. E. J. Obien, K. Deligkaris, T. Bullmann, D. J. Bakkum, and U. Frey, “Revealing neuronal function through microelectrode array recordings.," Frontiers in neuroscience, vol. 8, no. 193, p. 423, 2014. 
[40] J. Sandoe and K. Eggan, “Opportunities and challenges of pluripotent stem cell neurodegenerative disease models.," Nature Neuroscience, vol. 16, pp. 780-789, June 2013.

[41] P. G. Wilson and S. S. Stice, "Development and differentiation of neural rosettes derived from human embryonic stem cells.," Stem cell reviews, vol. 2, no. 1, pp. 67-77, 2006.

[42] Y. Elkabetz, G. Panagiotakos, G. Al Shamy, N. D. Socci, V. Tabar, and L. Studer, “Human ES cell-derived neural rosettes reveal a functionally distinct early neural stem cell stage.," Genes $\mathcal{E}$ Development, vol. 22, pp. 152-165, Jan. 2008.

[43] M. Castrén, T. Tervonen, V. Kärkkäinen, S. Heinonen, E. Castrén, K. Larsson, C. E. Bakker, B. A. Oostra, and K. Akerman, "Altered differentiation of neural stem cells in fragile X syndrome.," Proceedings of the National Academy of Sciences of the United States of America, vol. 102, pp. 17834-17839, Dec. 2005.

[44] Y. Luo, G. Shan, W. Guo, R. D. Smrt, E. B. Johnson, X. Li, R. L. Pfeiffer, K. E. Szulwach, R. Duan, B. Z. Barkho, W. Li, C. Liu, P. Jin, and X. Zhao, "Fragile $x$ mental retardation protein regulates proliferation and differentiation of adult neural stem/progenitor cells.," PLoS Genetics, vol. 6, p. e1000898, Apr. 2010.

[45] M. A. Callan, C. Cabernard, J. Heck, S. Luois, C. Q. Doe, and D. C. Zarnescu, “Fragile X protein controls neural stem cell proliferation in the Drosophila brain.," Human molecular genetics, vol. 19, pp. 3068-3079, Aug. 2010.

[46] L. N. Antar, C. Li, H. Zhang, R. C. Carroll, and G. J. Bassell, "Local functions for FMRP in axon growth cone motility and activity-dependent regulation of filopodia and spine synapses.," Molecular and cellular neurosciences, vol. 32, pp. 37-48, May 2006.

[47] A. Bhattacharyya, E. McMillan, K. Wallace, T. C. Tubon Jr, E. E. Capowski, and C. N. Svendsen, “Normal Neurogenesis but Abnormal Gene Expression in Human Fragile X Cortical Progenitor Cells," Stem cells and development, vol. 17, pp. 107-118, Feb. 2008.

[48] A. Oguro-Ando, A. Zuko, K. T. E. Kleijer, and J. P. H. Burbach, “A current view on contactin-4, -5, and -6: Implications in neurodevelopmental disorders.," Molecular and cellular neurosciences, vol. 81, pp. 72-83, June 2017.

[49] M. L. Montesinos, "Roles for DSCAM and DSCAML1 in central nervous system development and disease.," Advances in neurobiology, vol. 8, pp. 249-270, 2014.

[50] J.-R. Lee, "Protein tyrosine phosphatase PTPRT as a regulator of synaptic formation and neuronal development.," BMB reports, vol. 48, pp. 249-255, May 2015.

[51] S. W. Moore, M. Tessier-Lavigne, and T. E. Kennedy, "Netrins and their receptors.," Advances in experimental medicine and biology, vol. 621, no. Chapter 2, pp. 17-31, 2007.

[52] O. Peñagarikano, B. S. Abrahams, E. I. Herman, K. D. Winden, A. Gdalyahu, H. Dong, L. I. Sonnenblick, R. Gruver, J. Almajano, A. Bragin, P. Golshani, J. T. Trachtenberg, E. Peles, and D. H. Geschwind, "Absence of CNTNAP2 leads to epilepsy, neuronal migration abnormalities, and core autism-related deficits.," Cell, vol. 147, pp. 235-246, Sept. 2011.

[53] G. R. Anderson, T. Galfin, W. Xu, J. Aoto, R. C. Malenka, and T. C. Südhof, “Candidate autism gene screen identifies critical role for cell-adhesion molecule CASPR2 in dendritic arborization and spine development.," PNAS, vol. 109, pp. 18120-18125, Oct. 2012.

[54] G. M. Barlow, X. N. Chen, Z. Y. Shi, G. E. Lyons, D. M. Kurnit, L. Celle, N. B. Spinner, E. Zackai, M. J. Pettenati, A. J. Van Riper, M. J. Vekemans, C. H. Mjaatvedt, and J. R. Korenberg, “Down syndrome congenital heart disease: a narrowed region and a candidate gene.," Genetics in Medicine, vol. 3, pp. 91-101, Mar. 2001.

[55] Y. Wang, W. Yan, J. Wang, Y. Zhou, J. Chen, B. Gu, and W. Cai, “Common genetic variants in GAL, GAP43 and NRSN1 and interaction networks confer susceptibility to Hirschsprung disease.," Journal of cellular and molecular medicine, vol. 22, pp. 3377-3387, July 2018.

[56] H. L. Archer, J. C. Evans, D. S. Millar, P. W. Thompson, A. M. Kerr, H. Leonard, J. Christodoulou, D. Ravine, L. Lazarou, L. Grove, C. Verity, S. D. Whatley, D. T. Pilz, J. R. Sampson, and A. J. Clarke, "NTNG1 mutations are a rare cause of Rett syndrome.," American journal of medical genetics Part A, vol. 140, pp. 691-694, Apr. 2006.

[57] K. J. Zaccaria, D. C. Lagace, A. J. Eisch, and J. S. McCasland, "Resistance to change and vulnerability to stress: autistic-like features of GAP43-deficient mice.," Genes, brain, and behavior, vol. 9, pp. 985-996, Nov. 2010.

[58] T. Fernandez, T. Morgan, N. Davis, A. Klin, A. Morris, A. Farhi, R. P. Lifton, and M. W. State, “Disruption of Contactin 4 (CNTN4) results in developmental delay and other features of 3p deletion syndrome.," American journal of human genetics, vol. 82, p. 1385, June 2008. 
[59] L. Ferron, “Fragile X mental retardation protein controls ion channel expression and activity.," The Journal of Physiology, vol. 594, pp. 5861-5867, Oct. 2016.

[60] X. Xu, C. Radulescu, K. Utami, and M. A. Pouladi, “Obtaining Multi-electrode Array Recordings from Human Induced Pluripotent Stem Cell-Derived Neurons," BIO-PROTOCOL, vol. 7, no. 22, 2017.

[61] R. Patro, G. Duggal, M. I. Love, R. A. Irizarry, and C. Kingsford, “Salmon provides fast and bias-aware quantification of transcript expression.," Nature methods, vol. 14, pp. 417-419, Apr. 2017.

[62] C. Soneson, M. I. Love, and M. D. Robinson, "Differential analyses for RNA-seq: transcript-level estimates improve gene-level inferences.," F1000Research, vol. 4, p. 1521, 2015.

[63] M. I. Love, W. Huber, and S. Anders, "Moderated estimation of fold change and dispersion for RNA-seq data with DESeq2.," Genome biology, vol. 15, no. 12, p. 550, 2014.

[64] R. C. Team, “R: A language and environment for statistical computing," 2013.

[65] G. Bindea, B. Mlecnik, H. Hackl, P. Charoentong, M. Tosolini, A. Kirilovsky, W.-H. Fridman, F. Pagès, Z. Trajanoski, and J. Galon, "ClueGO: a Cytoscape plug-in to decipher functionally grouped gene ontology and pathway annotation networks.," Bioinformatics (Oxford, England), vol. 25, pp. 1091-1093, Apr. 2009.

[66] S. Anders and W. Huber, “Differential expression analysis for sequence count data," Genome biology, vol. 11, no. 10, p. R106, 2010.

[67] R. S. Sokol and P. H. Sneath, "Principles of numerical taxonomy," 1963.

[68] T. F. Cox and M. A. Cox, Multidimensional scaling. Chapman and hall/CRC, 2000.

[69] M. R. Anderberg, “Cluster analysis for applications," tech. rep., 1973.

[70] P. E. Geyer, N. A. Kulak, G. Pichler, L. M. Holdt, D. Teupser, and M. Mann, "Plasma Proteome Profiling to Assess Human Health and Disease," Cell Systems, vol. 2, pp. 185-195, Mar. 2016.

[71] N. A. Kulak, G. Pichler, I. Paron, N. Nagaraj, and M. Mann, “Minimal, encapsulated proteomic-sample processing applied to copy-number estimation in eukaryotic cells," Nature methods, vol. 11, pp. 319-324, Feb. 2014.

[72] J. Cox and M. Mann, "MaxQuant enables high peptide identification rates, individualized p.p.b.-range mass accuracies and proteome-wide protein quantification," Nature biotechnology, vol. 26, pp. 1367-1372, Nov. 2008.

[73] J. Cox, M. Y. Hein, C. A. Luber, I. Paron, N. Nagaraj, and M. Mann, “Accurate Proteome-wide Label-free Quantification by Delayed Normalization and Maximal Peptide Ratio Extraction, Termed MaxLFQ," Molecular E cellular proteomics : MCP, vol. 13, pp. 2513-2526, Sept. 2014.

[74] C. Lazar, L. Gatto, M. Ferro, C. Bruley, and T. Burger, "Accounting for the Multiple Natures of Missing Values in Label-Free Quantitative Proteomics Data Sets to Compare Imputation Strategies.," Journal of Proteome Research, vol. 15, pp. 1116-1125, Apr. 2016.

[75] P. Shannon, A. Markiel, O. Ozier, N. S. Baliga, J. T. Wang, D. Ramage, N. Amin, B. Schwikowski, and T. Ideker, "Cytoscape: a software environment for integrated models of biomolecular interaction networks.," Genome research, vol. 13, pp. 2498-2504, Nov. 2003.

[76] A. Franceschini, D. Szklarczyk, S. Frankild, M. Kuhn, M. Simonovic, A. Roth, J. Lin, P. Minguez, P. Bork, C. von Mering, and L. J. Jensen, "STRING v9.1: protein-protein interaction networks, with increased coverage and integration," Nucleic Acids Research, vol. 41, pp. D808-D815, Nov. 2012.

[77] M. E. J. Newman and M. Girvan, "Finding and evaluating community structure in networks," Physical Review E, vol. 69, p. 268, Feb. 2004. 